\title{
DYNAMIQUE DES POPULATIONS ET PRODUCTION DU ZOOPLANCTON DU LAC DE PORT-BIELH (PYRENEES CENTRALES)
}

\author{
par J. REY ${ }^{1,2}$ et J. CapblancQ ${ }^{1}$.
}

Trois Crustacés et quatre Rotifères composent le zooplancton du lac de Port-Bielh $(2285 \mathrm{~m})$ où dominent Mixodiaptomus laciniatus et Daphnia longispina.

La distribution verticale des organismes et ses variations nycthémérales diffèrent selon les espèces et, pour une même espèce, selon les stades de développement. La quantité d'animaux exportés en $24 \mathrm{~h}$ par le déversoir, liée à la distribution verticale du zooplancton, représente un faible pourcentage des populations $(0,14$ à $2,8 \%$ ) mais une biomasse importante $(0,45$ à $1,2 \mathrm{~kg}$ PF/jour).

Le cycle de développement des Crustacés se caractérise par une longue phase de repos durant les 7 mois de gel, une courte période de reproduction aú début de l'été et une phase de croissance rapide (août et septembre) aboultissant à une biomasse maximale en octobre. La durée de développement des différents stades de $M$. laciniatus et de $I$. longispina, déterminée d'après l'évolution numérique des populations, est proche des durées obtenues expérimentalement par plusieurs auteurs.

La production de $M$. laciniatus et de $D$. Longispina a été calculée à yartir de l'évolution du poids des individus en fonction de leur âge et de la variation du poids moyen des organismes au cours de l'été. Ces deux méthodes et leur variante graphique donnent des résultats assez voisins. Le rapport Production/Biomasse maximale est comparable pour les deux espèces $(1,35$ à 1,65 ) et se situe dans les valeurs déjà mentionnées pour des populations d'insectes aquatiques d'altitude. La production du Rotifère dominant (I'olyartra vulgaris) représente moins de $1 \%$ de celle des deux Crustacés.

La comparaison de la production primaire et secondaire de la zone pélagique indique une exploitation intense du phytoplancton par les herbivores. Malgré une faible biomasse, les algues constituent vraisemblablement une source de nourriture suffisante pour assurer la production du zooplancton.

\section{Population dynamics and production of the zooplankton of Lake Port-Bielh.}

Three species of Crustacea and four species of Rotifera form the zooplankton of Lake Port-Bielh (altitude $2285 \mathrm{~m}$ ) and the dominant species are Mixodiaptomus laciniatus and Daphnia longispina. The vertical distribution and diel variations of the organisms vary according to species and according to stages of development for the same species. The quantity of animals leaving the lake through the outflow in $24 \mathrm{~h}$ is linked to the vertical distribution of the zooplankton, and represents a small percentage of the population (0.14-2.8\%o) but forms an important biomass $(0.45-1.2 \mathrm{~kg}$ wet weight/day).

1. Laboratoire d'Hydrobiologie, Université Paul-Sabatier, 118, route de Narbonne, 31077 Toulouse Cedex.

2. Ce mémoire constitue une partie d'une thèse présentée par $\mathbf{M}^{m \text { " J }}$. Rey à l'Université Paul-Sabatier de Toulouse, en vue de l'obtention du Doctorat ès Sciences Naturelles. 
The development eycle of the Crustacea is typically a long resting period during the 7 months of ice, a short period of reproduction at the beginning of summer, and a period of rapid growth (August to September) with maximum biomass in Oetober. The length of development for the different stages of $M$. laciniatus and $D$. Iongispina, determined from the numerical evolution of the population, is similar to the lengths or development obtained experimentally by several authors. The production of $M$. laciniatus and $l$. longispina has been calculated from the changes in the weight of individuals as a function of their age, and from the variations in the mean weight of the organisms during the summer. The two methods gave very similar results. The ratio Production/Maximum Biomass is similar for the two species (1.35-1.65) and is similar to values already given for aquatic insects at this altitude. The production of the dominant species of Rotifera (Polyarthra vulgaris) represents less than $1 \%$ of that of the two species of Crustacea.

A comparison of primary and secondary production in the pelagic zone shows that there is an intense exploitation of phytoplankton by the herbivores; in spite of their low biomass, the algae probably form a food souree which is sufficient to ensure the production of the zooplankton.

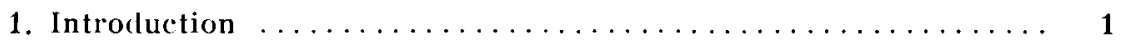

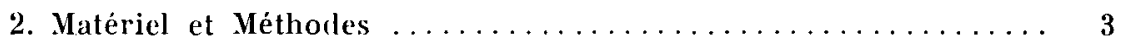

3. Etude du peuplement zooplanctonique

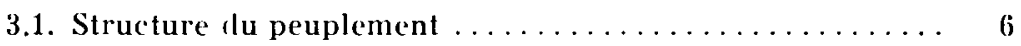

3.2. Migrations verticales $\ldots \ldots \ldots \ldots \ldots \ldots \ldots \ldots \ldots \ldots \ldots$

3.3. Dérive dans le déversoir $\ldots \ldots \ldots \ldots \ldots \ldots \ldots \ldots \ldots \ldots 14$

4. Cycles biologiques $\ldots \ldots \ldots \ldots \ldots \ldots \ldots \ldots \ldots \ldots \ldots \ldots \ldots \ldots$

5. Biomasse et Production

5.1. Paramètres de base et méthorles d'évaluation ....... 20

5.2. Mixodiaptomus laciniatus $\ldots \ldots \ldots \ldots \ldots \ldots \ldots \ldots \ldots$

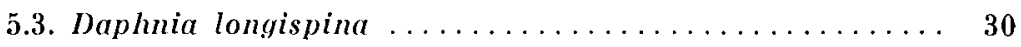

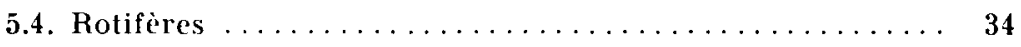

5.5. Comparaison des méthodes d'évaluation de la Produc-

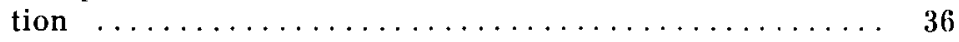

5.6. Biomasse et Production totale $\ldots \ldots \ldots \ldots \ldots \ldots \ldots, 40$

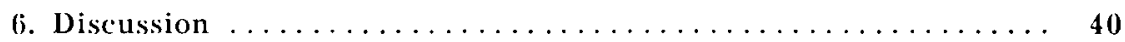

\section{1. - INTRODUCTION}

Situé dans le massif granitique du Néouvielle à une altitude de $2285 \mathrm{~m}$, le lac de Porl-Bielh présente les caractéristiques générales des lacs alpins : eaux froides transparentes el faiblement minéralisées, alternance d'une longue phase de gel ot d'une courte saison estivale. Les propriétés physico-chimiques, la faune benthique, la productivité primaire pélagique et benthique ont déjà fait l'objet de plusieurs travaux effectués dans le cadre du Programme Biolo- 
gicue International (Capblance 1972, 1973 ; Capblancq el Laville 1968, 1972 ; Bonnel et Capblance 1973 ; Laville 1972 a et b, 1974 ; Rey el Dupin 1973 a el b ; Giani et Laville 1973; Juget et Giani 1974 ; Giani et Lucas 1974).

Le présent travail porte sur le peuplement zooplanctonique dont nous avons étudié la dynamique des populations et la productivilé. Les résultats sont basés sur une campagne d'échanlillonnages réalisés au cours de l'été 1971 . La stabilité des conditions de milieu el du peuplement déjà mise en évidence pour le phytoplancton (Capblancq 1972) et les invertébrés benthiques (Laville 1972), permet de penser que ces résulta!s sont représentatifs de la moyenne du milieu.

\section{2. - MATÊRIEL ET MÉTHODES}

L'échantillonnage a été réalisé du 29 juin (moment du dégel) au 5 novembre 1971, à la fréquence d'un relevé par semaine. Trois prélèvement hivernaux (20-II, 30-III, 2-VI) complètent cette série de relevés. Deux méthodés ont été ulilisées simultanément :

- prélèvements de 5 litres d'eau effectués avec une bouleille Van Dorn, aux profondeurs de $0,5,10,15$ et $18 \mathrm{~m}$, dans deux stations situées au niveau de's deux fosses du lac. Les organismes ont été concentrés sur filet fin (vide de maille de $50 \mu$ ) el fixés par quelques gouttes de formol ;

- caplure dorganismics au moven d'un filet à plancton remonté de $18 \mathrm{~m}$ jusqu'à la surface.

Après sédimentation dans des chambres combinées, les échantillons onl été comptés à l'aide d'un microscope inversé pour les peliles formes (Rotifères, slades naupliens) el d'une loupe binoculaire $(\mathrm{G} 12,5 \times 4)$ pour les formes de plus grande taille (copépodites el Copépodes adultes, Cladocères). Dans ces comptages, nous avons dénombré séparément les individus des divers stades de développement des Copépodes ; Daphnia longispina a été complabiliséc en distinguant $q$, $q$ ovigères, $q$ éphippiales et $\hat{\delta}$. Dans chacune de ces catégories, nous avons mesuré 20 à 100 individus issus du matériel récolté au filet. Ce matériel a également servi à évaluer, d'une part le nombre moyen d'oufs portés plar les $q$ ovigères de D. longispina et Mixodiaptomus laciniatus, d'autre part la proportion de $q$ ovigères chez les Rotifères.

En outre, nous avons réalisé au cours de l'été deux séries de prélèvements sur un cycle de $24 \mathrm{~h}$, afin d'analyser les migrations verticales des organismes. Un filet de Clarke Bumpus placé en même temps dans le déversoir du lac el relevé loutes les 2 heures, a permis de déterminer le nombre d'organismes en dérive pendant

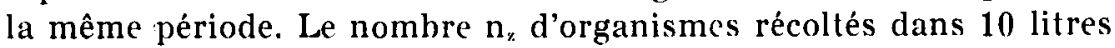


d'eau aux profondeurs retenues, nous a permis de calculer l'effectif moyen de chaque stade dans la colonne d'eau $0 \mathrm{~m}-18 \mathrm{~m}$ :

$$
\overline{\mathrm{N}} / \mathrm{m}_{3}=\frac{100}{18}\left(\frac{\mathrm{n}_{0}+\mathrm{n}_{5}}{2} \times 5\right)+\left(\frac{\mathrm{n}_{5}+\mathrm{n}_{10}}{2} \times 5\right)+\left(\frac{\mathrm{n}_{10}+\mathrm{n}_{15}}{2} \times 5\right)+\left(\frac{\mathrm{n}_{15}+\mathrm{n}_{18}}{2} \times 3\right)
$$

La variation de $\overline{\mathrm{N}}$ entre relevés parallèles a été testée lors des séries de prélèvements réalisés sur un cycle de $24 \mathrm{~h}$. Les résultats figurent dans le tableau I. Dans tous les cas, les valeurs du $\chi^{2}$ sont en accord avec l'hypothèse d'une distribution au hasard, et l'intervalle de confiance de la moyenne est inversement proportionnel à la densité de la population dans le lac. L'échantillonnage ayant toujours été réalisé de la même façon $(2 \times 5$ litres d'eau à chaque profondeur), la marge d'erreur des estimations de l'effectif moyen dépend de la densité des organismes; elle sera d'autant moins forte que le nombre d’organismes présents dans le milieu sera plus élevé.

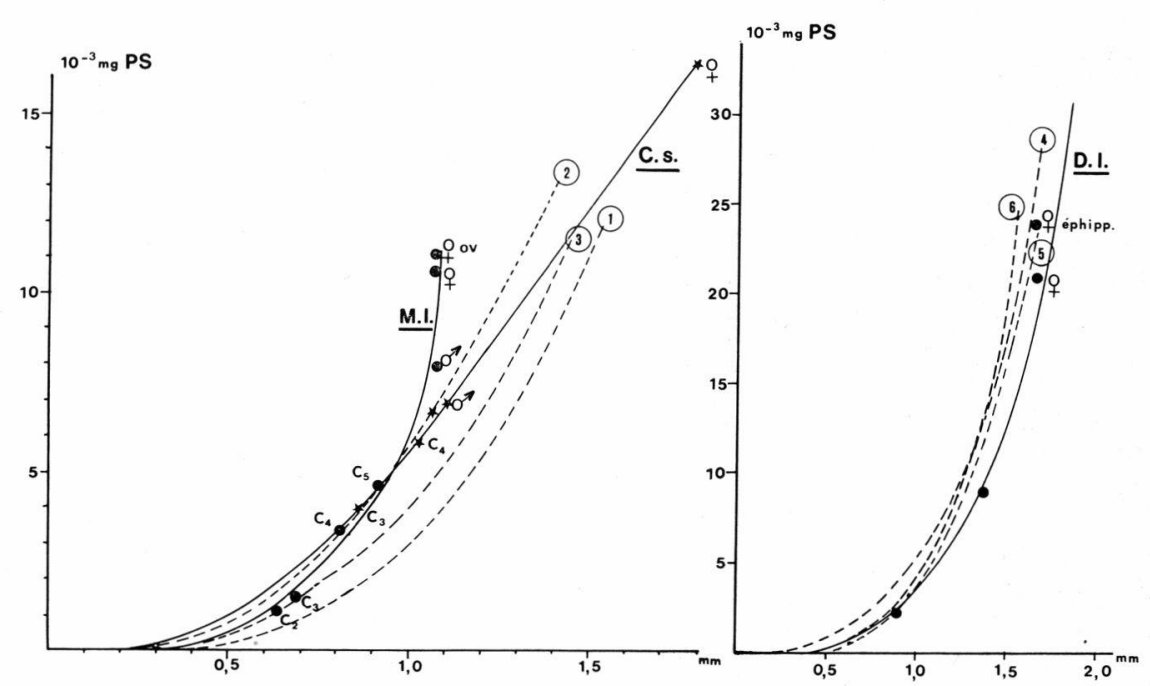

FIG. 1. - Relation taille-poids chez Mixodiaptomus laciniatus (M.1.) : $\mathrm{P}_{\mathrm{mg}} \mathrm{PS}=$ $0,00714 \mathrm{~L}_{\mathrm{mm}}^{3,8}$, Cyclops strenuus (C.s.) : $\mathrm{P}_{\mathrm{mg}} \mathrm{PS}=0,006 \mathrm{~L}_{\mathrm{mm}}^{2,44}$, Daphnia longispina (D.l.) : $\mathrm{P}_{\mathrm{mg}} \mathrm{PS}=0,0031 \mathrm{~L}_{\mathrm{mm}}^{3,56}$. En tirets : relations établies pour des Copépodes et des Cladocères par: Scherbakov (1952 (1), Klekowski et Sushkina 1966 (2), Mordukai-Boltovskoy 1954 (3 et 4), Pecen 1965 (5), Osmera 1966 (6).

Les relations taille-poids (fig. 1) ont été établies à partir de pesées de lots d'individus $(200$ à 500$)$ regroupés par stade ou par classe de taille, après séchage à l'étuve à $60^{\circ}$ pendant $48 \mathrm{~h}$. Les pesées de zooplancton après essorage sur papier filtre (Poids frais) et après dessication à l'étuve (Poids sec), confirment la stabilité du 
(5)

ZOOPLANCTON DU LAC DE PORT-BIELH

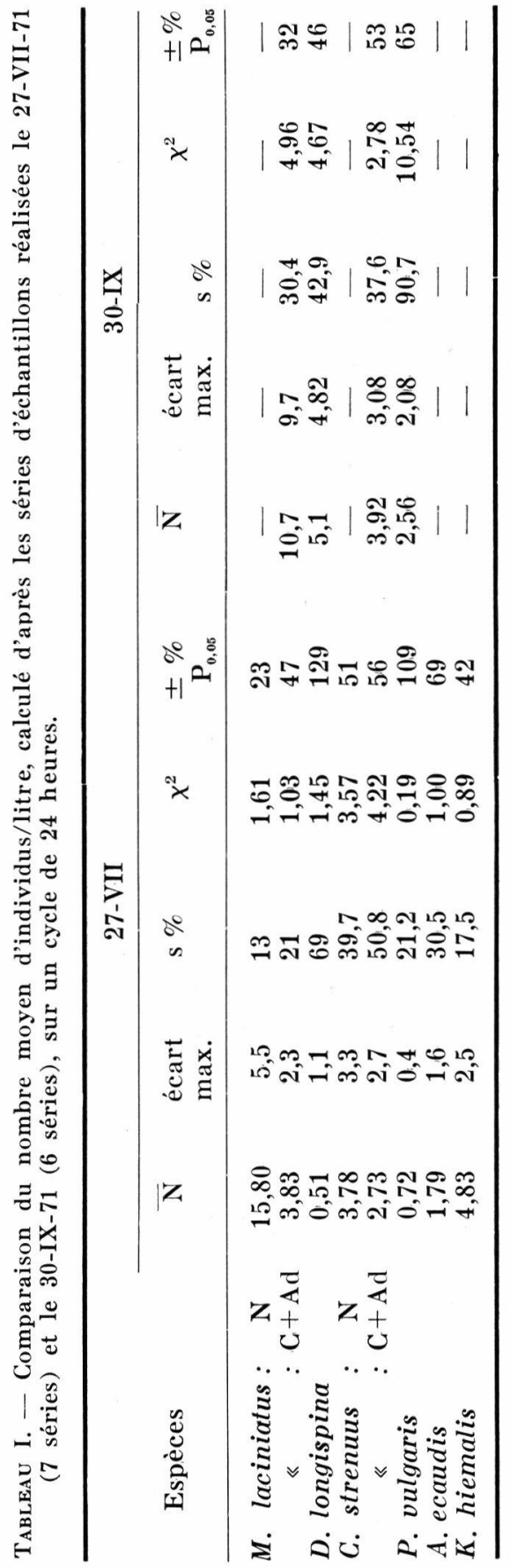


rapport $\frac{P S}{P F}=0,11$, valeur très proche de celle de 0,10 communément admise (Winberg et coll. 1971).

Le poids des œufs de $M$. laciniatus et $D$. longispina a été obtenu à partir de la différence de poids entre les $q$ et les $q$ ovigères d'une part, et par estimation du biovolume d'un œuf d'autre part. Si l'on admet un rapport $\mathrm{PS} / \mathrm{PF}=\mathbf{0 , 1}$, ces deux méthodes ont fourni des résultats comparables, ainsi que le montrent les chiffres suivants :

\begin{tabular}{lcl}
\hline & Estimation par biovolume & $\Delta \mathrm{P}$ \\
\hline$\omega$ de $M$. laciniatus & $0,18 \mu \mathrm{g}$ PS & $0,15 \mu \mathrm{g}$ PS \\
$\omega$ de $D$. longispina & $0,8 \mu \mathrm{g}$ PS & $0,5 \mu \mathrm{g}$ PS \\
\hline
\end{tabular}

\section{3. - ETUDE DU PEUPLEMENT ZOOPLANCTONIQUE}

\section{1. - Structure du peuplement.}

La zone pélagique du lac de Port-Bielh est colonisée par 3 espèces de Crustacés et 4 espèces de Rotifères :

Copépodes Mixodiaptomus laciniatus (Lilljeborg)

Cyclops strenuus Fischer

Cladocères Daphnia longispina O. F. Müller

Rotifères Polyarthra vulgaris $f$. aspina Pourriot

Keratella hiemalis Carlin

Conochilus unicornis Rousselet

Ascomorpha ecaudis Perty.

L'évolution numérique des populations de ces 7 espèces et leur distribution en profondeur ont été représentées dans les figures 3 à 9 . Les conditions thermiques sont schématisées par les courbes isothermes de la figure 2.

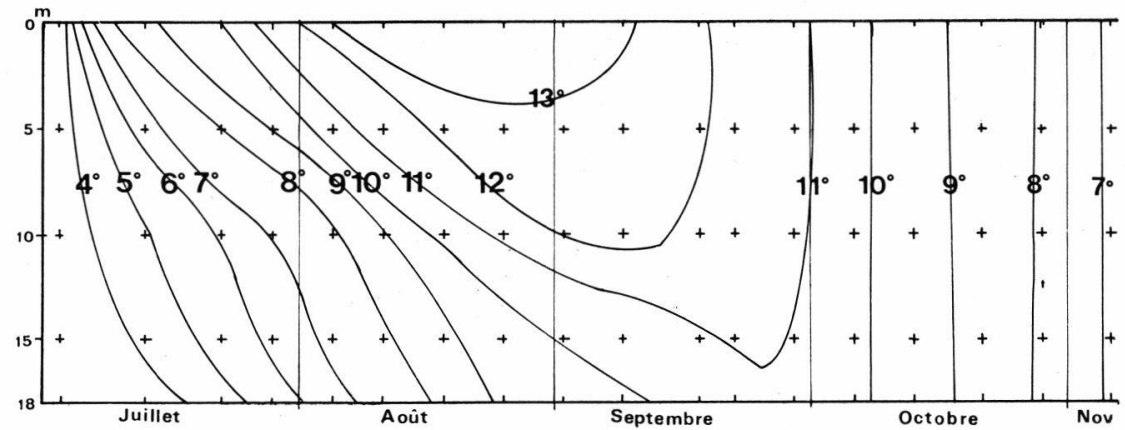

Fig. 2. - Courbes isothermes du lac de Port-Bielh en 1971, Les + indiquent les points de relevés. 
a) Copépodes.

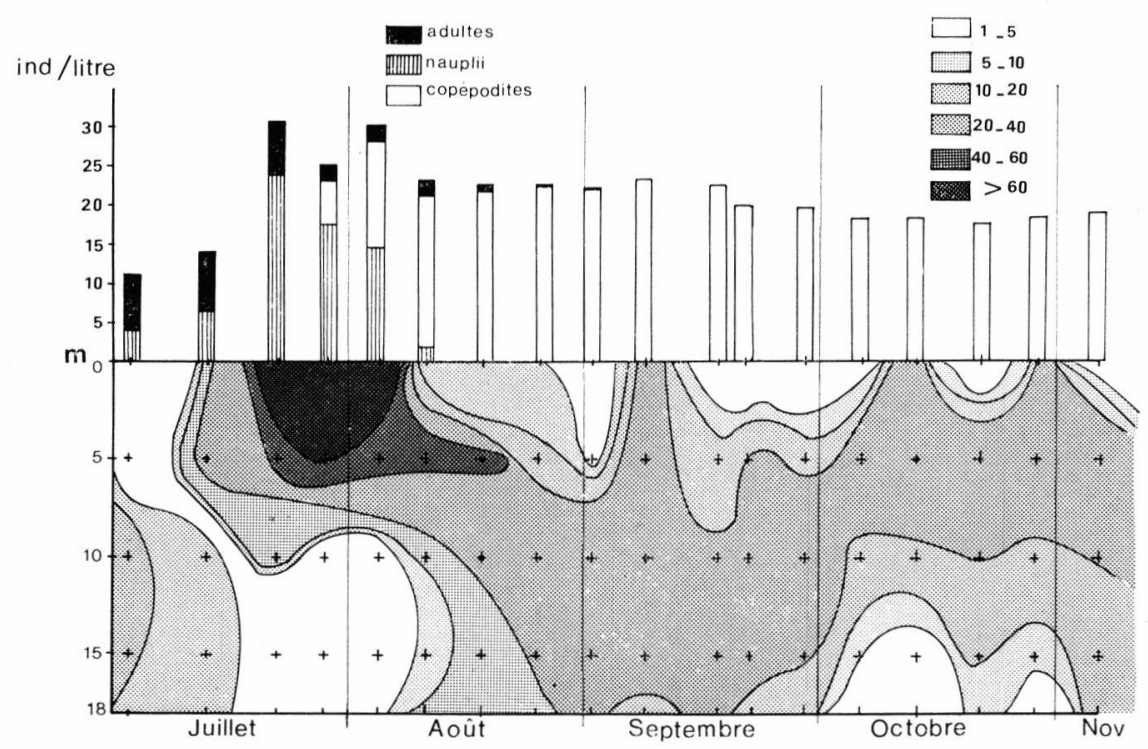

FIG. 3. - Mixodiaptomus laciniatus : Evolution du nombre moyen d'individus (histogrammes) et zones d'équidensité.

Mixodiaptomus laciniatus (fig. 3) est l'élément dominant du zooplancton du lac de Port-Bielh. Considérée comme une forme sténotherme d'eau froide, cette espèce est présente toute l'année. Sa densité moyenne varie de 8-9 individus/litre à la fin de l'hiver, l'espèce étant alors uniquement représentée par des copépodites $\mathrm{V}$ et des adultes, à 22-25 individus/litre à la fin du mois de juillet, époque où les nauplii constituent plus de $90 \%$ de la population.

La distribution des individus en profondeur varie au cours de l'été au fur et à mesure du développement des organismes et de l'évolution des profils thermiques.

En juillet, période de stratification thermique, les stades naupliens se concentrent dans la zone superficielle du lac. Entre le 12 juillet et le 4 août, 45 à $77 \%$ de la population présente dans la colonne d'eau $0 \mathrm{~m}-18 \mathrm{~m}$, évolue entre 0 et $6 \mathrm{~m}$. Au mois d'août, la stratification thermique s'atténuant, les stades copépodites I à III qui constituent l'essentiel de la population, se répartissent entre 5 et $15 \mathrm{~m}$, avec un maximum entre 10 et $15 \mathrm{~m}$.

Durant l'isothermie d'automne, les stades copépodites IV et V colonisent toute la masse d'eau avec, cependant, une densité moindre en surface $(0-5 \mathrm{~m})$ et dans la zone profonde $(15-18 \mathrm{~m})$, et un maximum dans la zone intermédiaire. L'étude du déplacement vertical des organismes (§ 3.2.) montre que les migrations journalières affectent peu cette distribution de la population. 


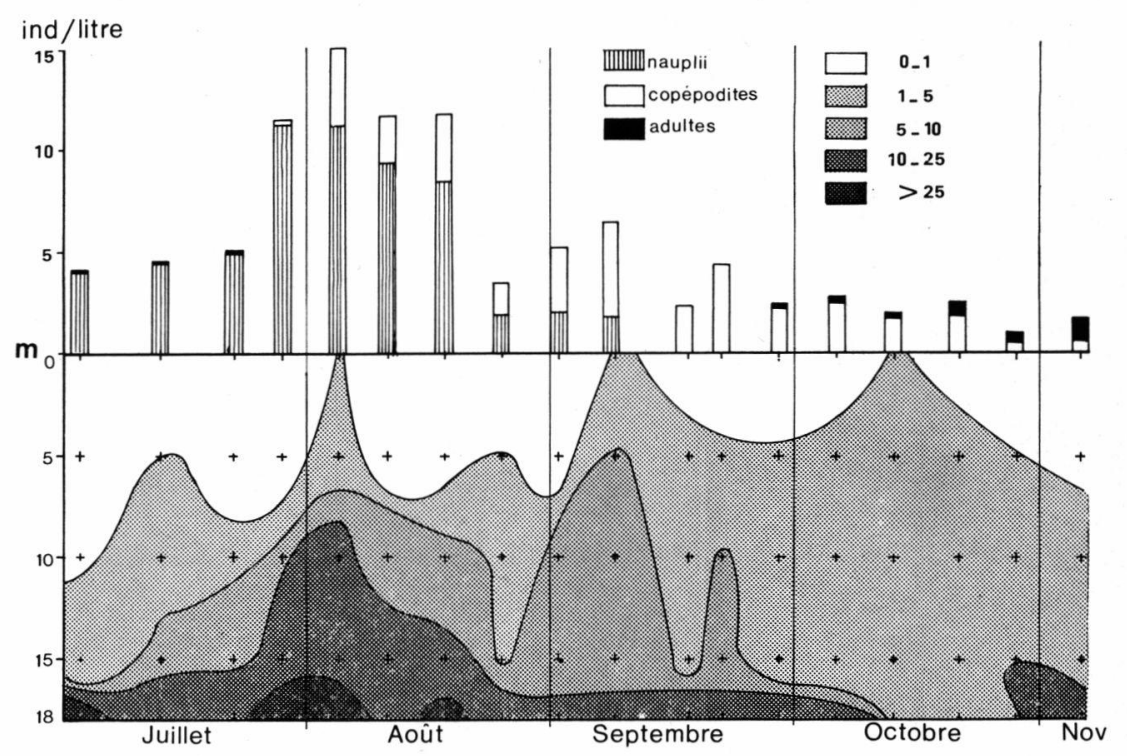

Fig. 4. - Cyclops strenuus : Evolution du nombre moyen d'individus (histogrammes) et zones d'équidensité.

Cyclops strenuus (fig. 4) est un des Copépodes dominant de la zone benthique profonde. Toutefois cette espèce n'est pas strictement benthique car elle colonise également la zone pélagique. Dans un lac voisin (Lac Long du vallon d'Estibère), Angelier (1961) et Gouxette (1966) ont montré que Cyclops strenuus pouvait remplacer les Diaptomides dans la niche écologique occupée par ces derniers dans les autres lacs du vallon.

Dans la zone pélagique du lac de Port-Bielh, cette espèce vit essentiellement dans les couches profondes $(15-18 \mathrm{~m})$ où elle paraît remplacer $M$. laciniatus. Les stades naupliens et copépodites jeunes abondent dans le plancton de profondeur, fin juillet-début août. Les stades copépodites IV, V et les adultes qui constituent l'essentiel de la population en automne, paraissent davantage inféodés à un mode de vie bentique et se rencontrent en densité moindre dans le plancton.

\section{b) Cladocères.}

Daphnia longispina (fig. 5) est la seule espèce peuplant la zone pélagique du lac de Port-Bielh.

Les premiers individus apparaissent à partir de la mi-juillet. La population présente ensuite deux maxima: le premier, fin août, est le fait de + parthénogénétiques ; le second, fin septembre, correspond au développement des ô et marque le début d'une phase 


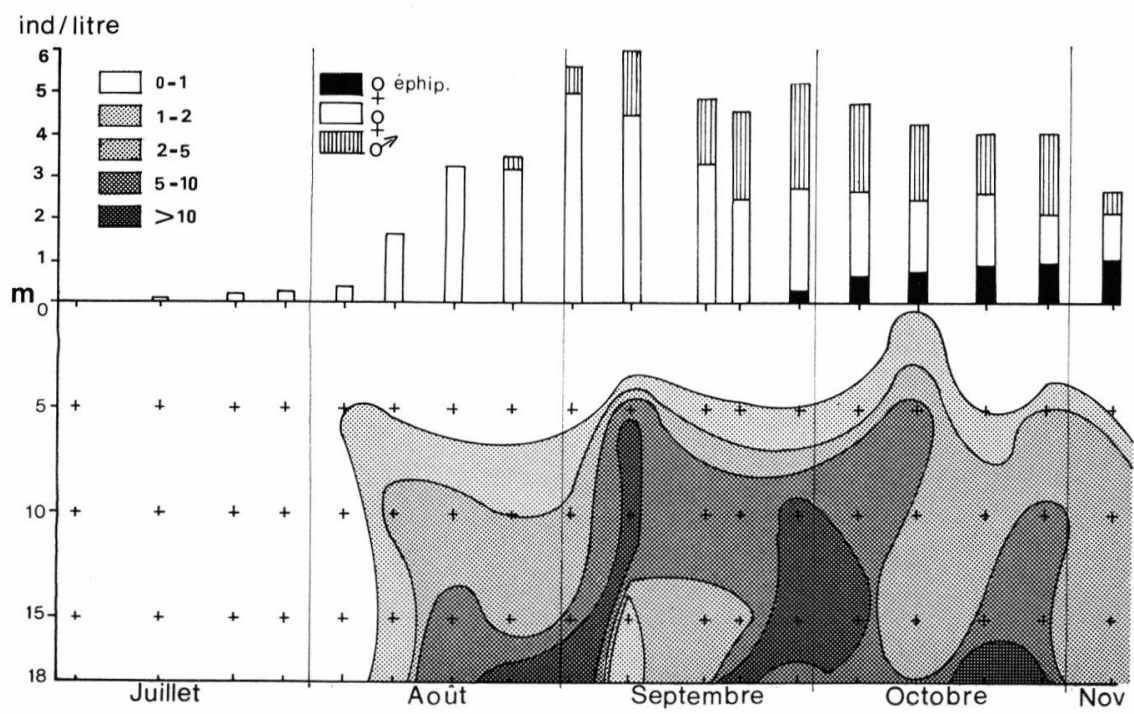

FIG. 5. - Daphnia longispina: Evolution du nombre moyen d'individus (histogrammes) et zones d'équidensité.

de reproduction sexuée. Le déclin de la population de ô à la fin du mois d'octobre, s'accompagne de l'augmentation du nombre des o éphippiales qui constituent le 5 novembre près de $50 \%$ des individus.

En juillet, D. Iongispina vit essentiellement en surface (60 à $80 \%$ de la population entre 0 et $10 \mathrm{~m}$ ). A partir du mois d'août, les plus fortes densités de cette espèce se rencontrent dans la zone profonde (60 à $90 \%$ de la population entre 10 et $18 \mathrm{~m}$ ).

\section{c) Rotifères.}

Polyarthra vulgaris (fig. 6) est le Rotifère planctonique dominant du lac de Port-Bielh. Il correspond à la forme aspina décrite par Pourriot (1965). Sa densité moyenne varie entre 0,2 ind./litre au dégel, et 30 ind./litre au début du mois de septembre. Pendant tout l'été, les plus fortes densités d'individus se situent au-delà de $10 \mathrm{~m}$ de profondeur, la couche $10-18 \mathrm{~m}$ renfermant généralement plus de $70 \%$ de la population.

Les trois autres Rotifères planctoniques (fig. 7, 8, 9) ont un développement plus éphémère et se succèdent au cours de l'été. Keratella hiemalis est une espèce sténotherme d'eau froide type. Elle peuple exclusivement la zone la plus profonde au début de l'été, tant que la température de l'eau n'excède pas $6^{\circ}$. Ascomorpha ecaudis puis Conochilus unicornis lui succèdent et atteignent une densité moyenne de 4 ind./litre le 10 août pour le premier, et de 10 ind./litre le $1^{\text {er }}$ septembre pour le second, avec un maximum situé à une profondeur de $10 \mathrm{~m}$ environ. 


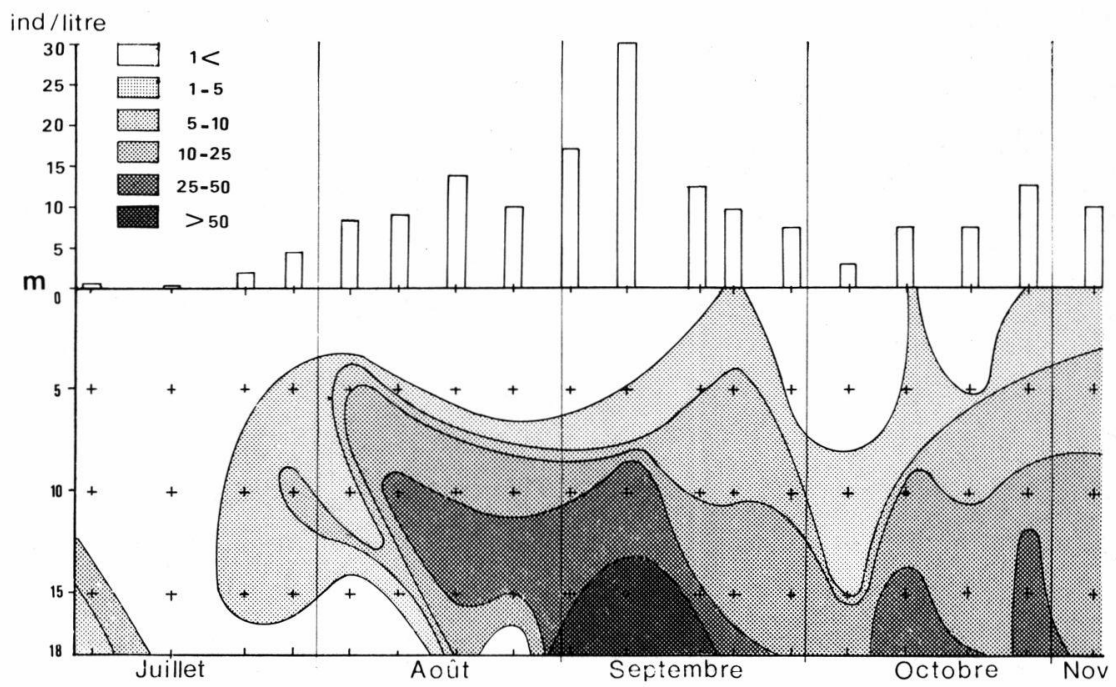

Fig. 6. - Polyarthra vulgaris : Evolution du nombre moyen d'individus (histogrammes) et zones d'équidensité.

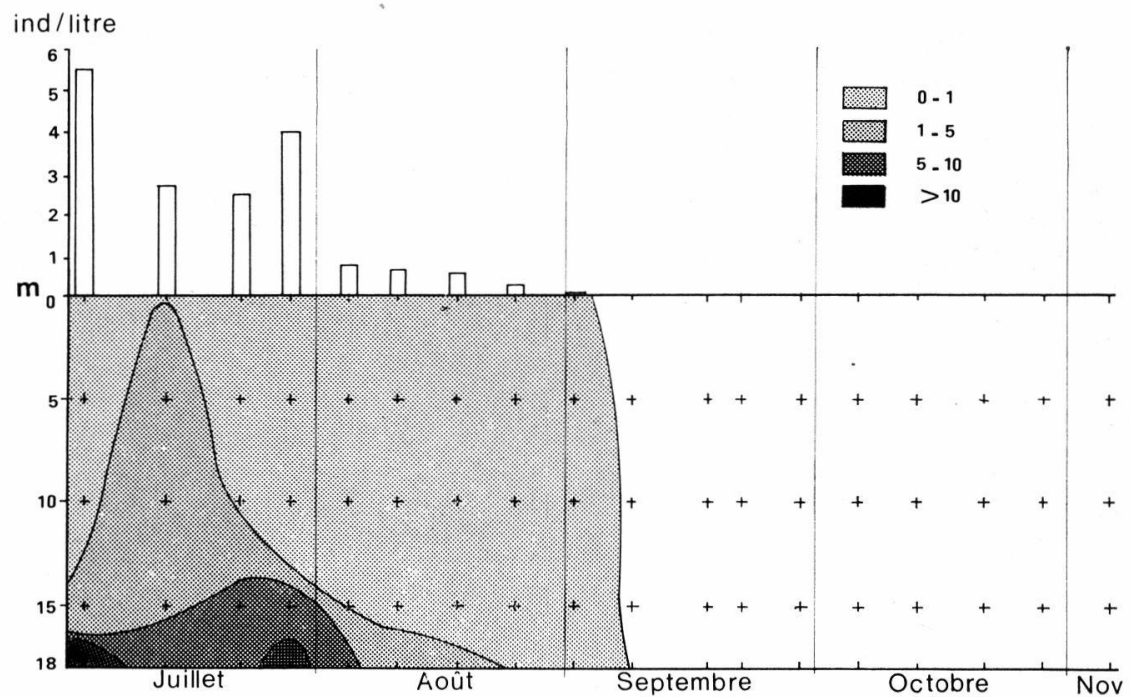

Fig. 7. - Keratella hiemalis : Evolution du nombre moyen d'individus (histogrammes) et zones d'équidensité. 


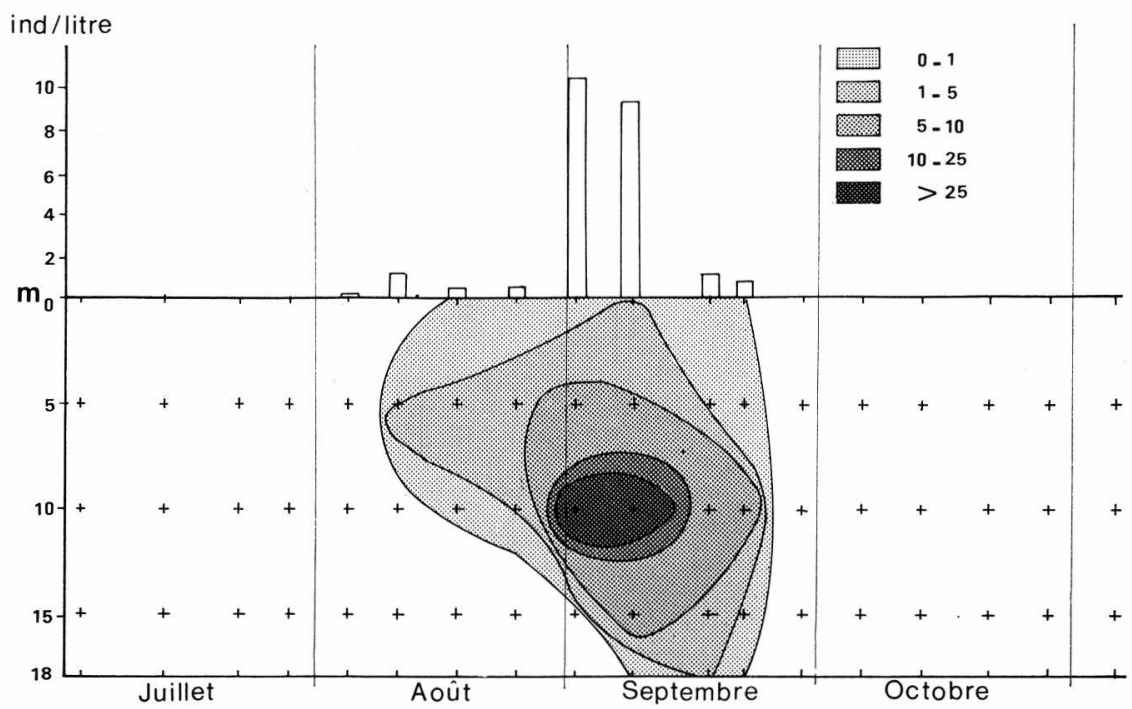

Fig. 8. - Conochilus unicornis : Evolution du nombre moyen d'individus (histogrammes) et zones d'équidensité.

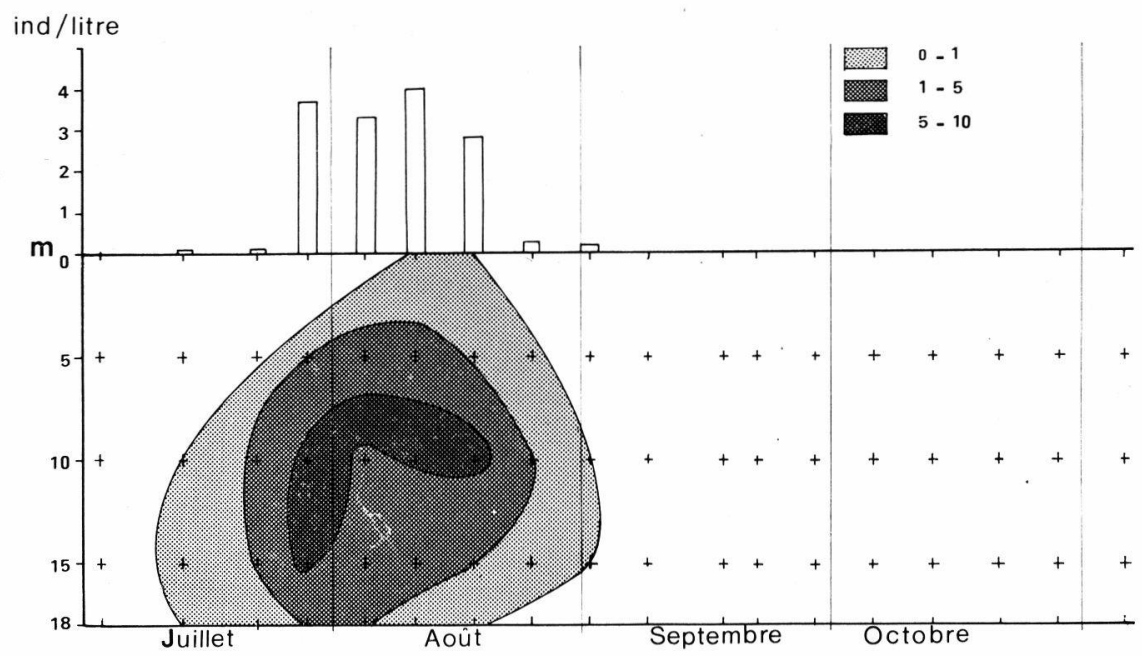

Fig. 9. - Ascomorpha ecaudis : Evolution du nombre moyen d'individus (histogrammes) et zones d'équidensité.

\section{2. - Migrations verticales.}

Les variations de la distribution du zooplancton au cours d'un cycle de $24 \mathrm{~h}$ ont été étudiées à 2 périodes, l'une correspondant à la phase de stratification thermique (août), l'autre à la phase 

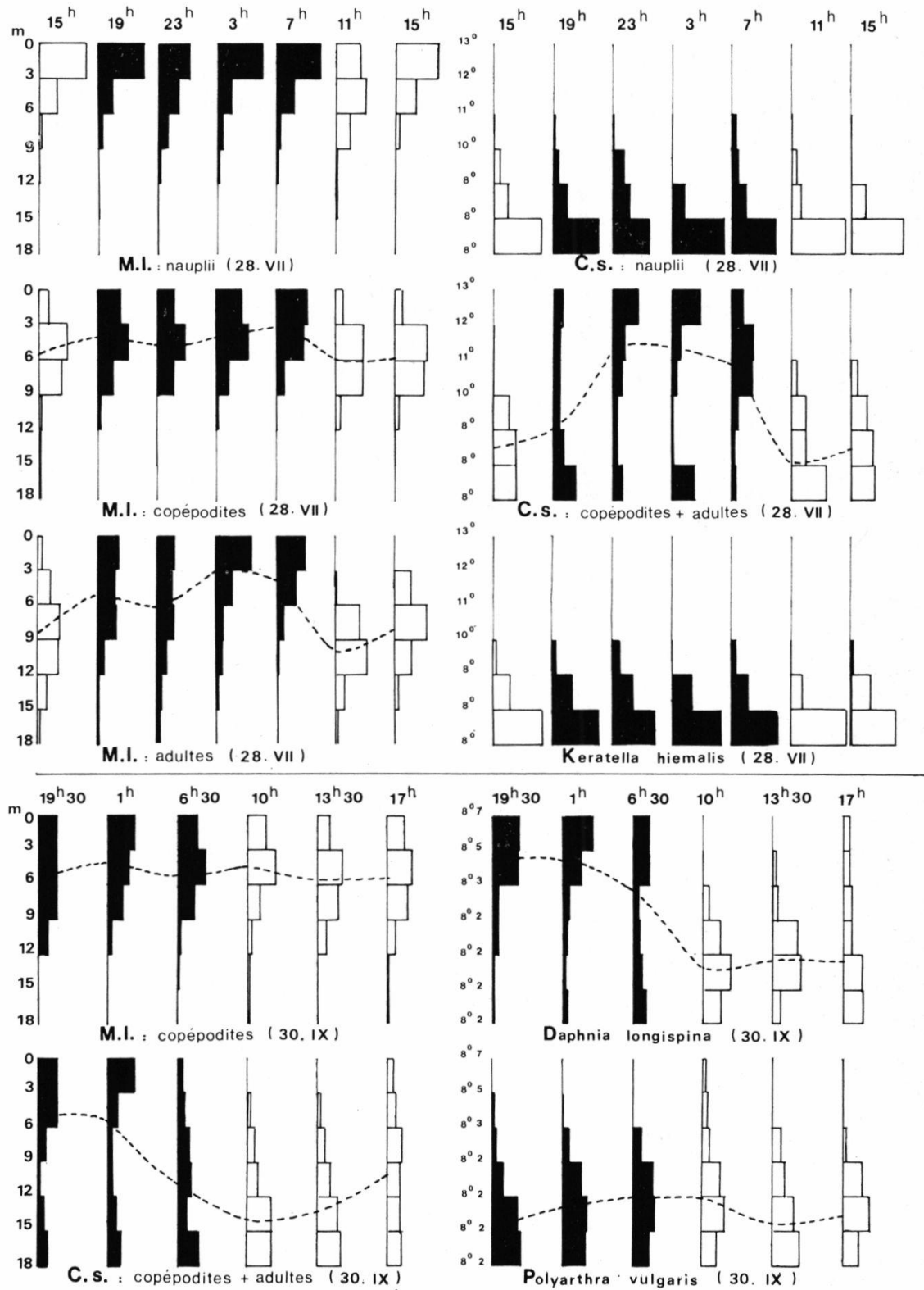

FIg, 10. - Répartition verticale des éléments dominants du zooplancton en période de stratification thermique ( 28 juillet), et d'isothermie automnale (30 septembre). Les histogrammes représentent la proportion d'individus présents dans des strates de $3 \mathrm{~m}$ de profondeur; les traits en pointillés indiquent la limite de $50 \%$ de la population.

M.1.: Mixodiaptomus laciniatus; C.s. : Cyclops strenuus. 
d'isothermie d'automne. Les résultats ont été représentés par des hislogrammes (fig. 10) pour différentes heures de la journée.

Au mois d'août, la population de Mixodiaptomus laciniatus est presque exclusivement composée de stades naupliens. Les nauplii se maintiennent dans la zone supérieure du lac, la tranche $0-6 \mathrm{~m}$ renfermant 76 à $93 \%$ des individus présents entre 0 et $18 \mathrm{~m}$. Les stades copépodites présentent des migrations verticales de faible amplitude : pendant la nuit 70 à $80 \%$ des copépodites se rencontrent entre 0 et $6 \mathrm{~m}$, contre 50 à $60 \%$ durant le jour.

La migration en profondeur est beaucoup plus nette pour les stades adultes : moins de $20 \%$ des individus entre 0 el $6 \mathrm{~m}$ le jour, pour 40 à $70 \%$ pendant la nuit.

Ces résultats présentent une analogic avec la distribution de la population de $M$. laciniatus en été, dont les nauplii sont concentrés en surface début août, et dont les stades copépodites colonisent les zones les plus profondes fin août et septembre.

En période d'isothermie, la population se répartit à peu près uniformément entre 0 et $15 \mathrm{~m}$ lout au long de la journée. Les histogrammes révèlent cependant une tendance à l'enfoncement des stades copépodites vers le milieu de la journée $(13 \mathrm{~h} 30)$. Dans tous les cas, la population décline fortement au-delà de $12 \mathrm{~m}$ de profondeur, les 6 derniers mètres de la colonne d'eau renfermant toujours moins de $15 \%$ de la population. $M$. laciniatus est remplacé ici par Cyclops strenuus avec lequel il semble entrer en concurrence.

Les stades naupliens de Cyclops strenuus se maintiennent dans la zone la plus profonde (plus de $50 \%$ de la population dans les 3 derniers mètres). Les stades plus âgés présentent par contre des mouvements de grande amplitude avec remonlée des organismes près de la surface la nuit (plus de $50 \%$ des adultes et des copépodites entre 0 et $6 \mathrm{~m}$ ) et un enfoncement diurne net (plus de $50 \%$ entre 12 et $18 \mathrm{~m}$ ).

Daphnia longispina présente un rythme nycthéméral de répartition caractérisé par une concentration diurne de la population dans les couches profondes, et une remontée nocturne des individus en surface. L'intégration des courbes de distribution verticale permet d'évaluer le nombre moyen d'individus par $\mathrm{m}^{3}$ entre 0 et $18 \mathrm{~m}$, à 3200 pendant la nuit, et à 7700 entre 11 h et 15 h. Cette estimation traduit ce phénomène de concentration des Cladocères dans les zones de plus grande profondeur, durant le jour.

La distribution des Rotifères (Polyarthra vulgaris, Keratella hiemalis, Ascomorpha ecaudis) ne présente aucune variation journalière notable.

En conclusion, il semble que les migrations des organismes, induites par les variations d'éclairement, soit liées à la taille des animaux (c'est-à-dire à leur plus ou moins grande mobilité), au moins en ce qui concerne l'amplitude des déplacements. Les petites 
Tableau II. - Dérive des organismes zooplanctoniques au déversoir du lac de Port-Bielh. Composition du peuplement en \% du peuplement total. Nombre total d'individus et poids frais avec limites de confiance à $P$ 0,05.

(a) : 1-2 juillet 71 ,

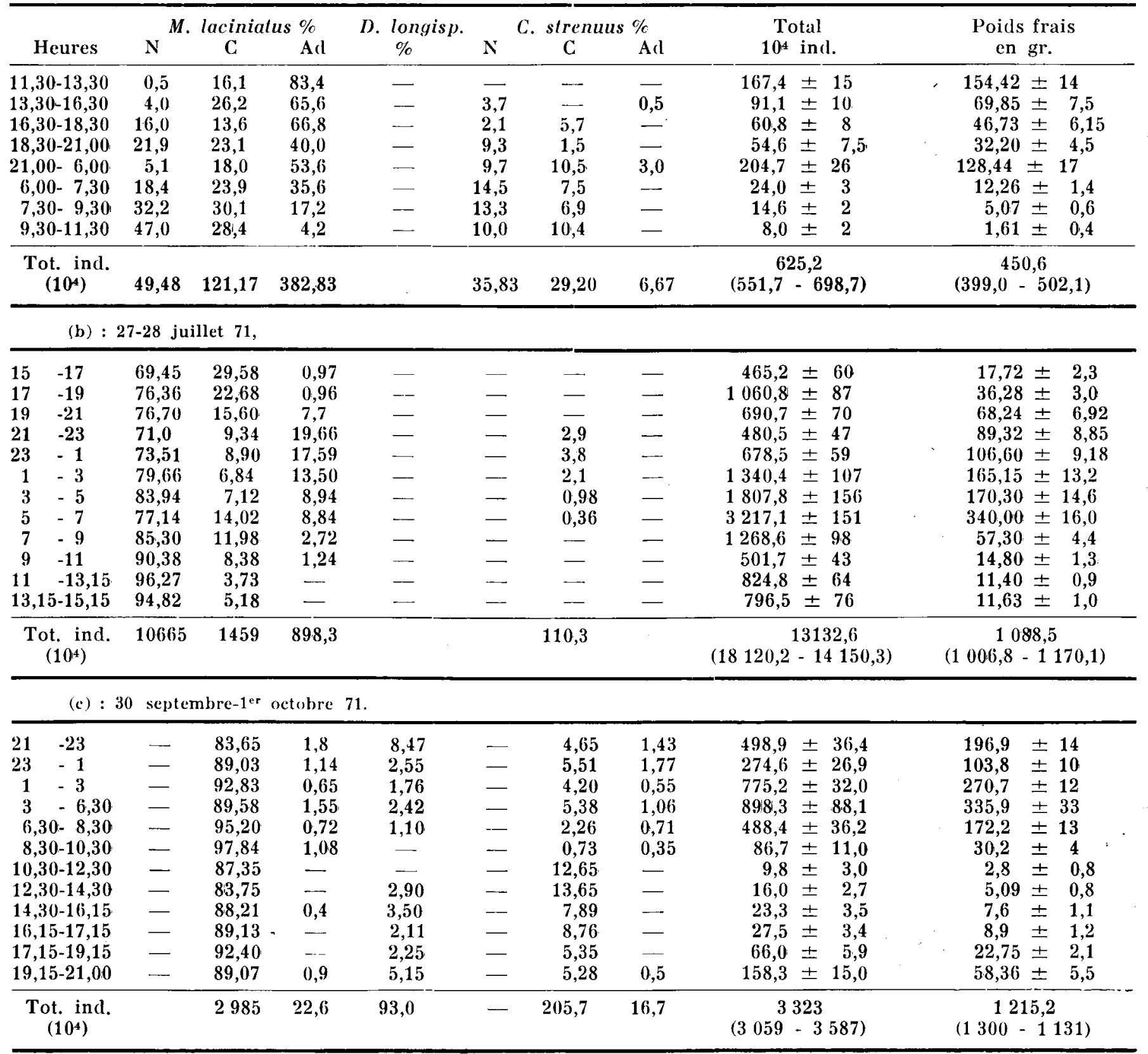


formes (Rolifères, slades naupliens, stades copépodites jeunes) présentent des mouvements de faible amplitude au cours d'un cycle de $24 \mathrm{~h}$; les organismes bon nageurs (D. longispina, adultes de $M$. laciniatus, subadultes et adultes de $C$. strenuus) présentent des migrations verticales plus amples, avec deseente de la population le jour et remonlée en surface la nuit. L'existence d'un gradient de température entre la surface et la profondeur, ne paraît pas influer sur ce phénomène.

\section{3. - Dérive du zooplancton dans le déversoir.}

La filtration d'une partie de l'eau s'écoulant par le déversoir a été pratiquée sur trois cycles de $24 \mathrm{~h}$, dans le but :

- d'établir une relation entre la nature et la quantité des organismes exportés et leur distribution verticale ;

- de caculer la quantité de zooplancton rejetée hors de l'écosystème et apportée au torrent dont le déversoir est la source.

Les résultats figurent dans le tableau II a, b, c. Leur examen amène les constatations suivantes :

a) La quantité d'organismes zooplancloniques exportés chaque jour par le déversoir est considérable. L'enregistrement de la quantité d'eau filtrée par le filet, et le larage du débit du déversoir lors de chaque expérience, ont permis d'évaluer cette quantité exportée à 6 millions d'ind. $/ 24 \mathrm{~h}$ au dégel, et 131 millions $/ 24 \mathrm{~h}$ fin juillet

La biomasse correspondante est de $450 \mathrm{gr} \mathrm{PF} / 24 \mathrm{~h}$ au dégel, et de $1088 \mathrm{gr}$ à $1125 \mathrm{gr} \quad \mathrm{PF} / 24 \mathrm{~h}$ respectivement début août - fin septembre.

Cette dérive du zooplancton est donc un phénomène non négligeable dans la mesure où ces organismes constituent une source notable de matières organiques pour les communautés benthiques du torrent issu du déversoir.

b) L'élément dominant des organismes en dérive est toujours Mixodiaptomus laciniatus. Par rapport à la population présente dans le lac, la perte quotidienne de cette espèce, de l'ordre de $0,28 \%$ en juillet et de $0,13 \%$ en septembre, constitue une hémorragie représentant de 25 à $50 \%$ de la mortalité journalière de la population.

Cyclops strenuus et Daphnia longispina sont exportés en quantité moindre. Le 30 septembre, le nombre de Daphnia perdues par le déversoir correspond à $0,14 \%$ de la population présente dans le lac.

c) Pour toutes les espèces, la dérive nocturne est supérieure à la dérive diurne. Plus de $80 \%$ de la biomasse transportée en $24 \mathrm{~h}$ début août et fin septembre, dérive pendant les heures de nuit.

Ce phénomène est le résultal de la migration des différentes 
espèces vers les couches supérieures du lac durant la nuit. La relation devient particulièrement nette à l'examen de la faune en dérive. Ainsi, le 9 août, les adultes et les stades copépodites de $M$. laciniatus représentent en moyenne $23 \%$ du nombre d'organismes recueillis pendant la nuit, $146 \%$ seulement pendant le jour. Les adultes de $C$. strenuus ne sont présents que dans les relevés de nuit. Le 30 septembre, C. strenuus et D. longispina relativement abondants pendant la nuit $\left(960\right.$ Cyclops, 560 Daphnia $/ \mathrm{m}^{3}$ en moyenne), n'apparaissent qu'à de rares exemplaires durant le jour (120 Cyclops et 37 Daphnia/ $\mathrm{m}^{3}$ en moyenne).

La remontée de la faune dans les couches de surface la nuit s'accompagne d'une augmentation de leur nombre dans l'eau du déversoir. La quantité et la nature de la faune en dérive est donc liée à la distribution verticale des organismes et à leurs migrations. La variation du nombre et de la biomasse des organismes exportés en $24 \mathrm{~h}$, et l'évolution du débit du déversoir au cours de l'été sont schématisées dans la figure 11.

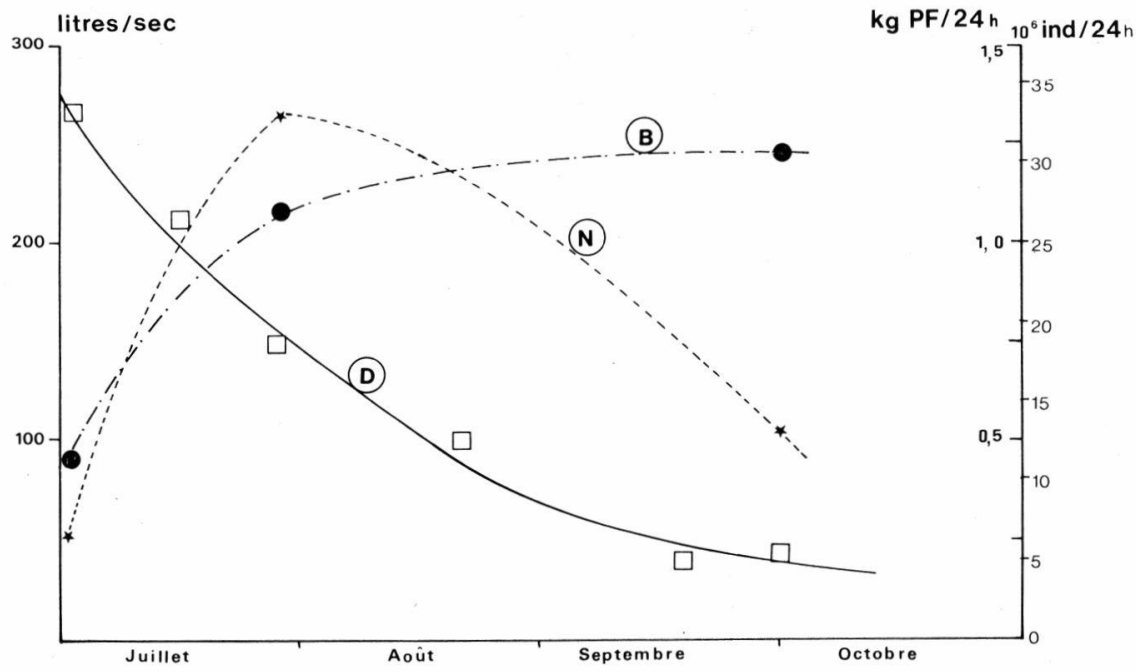

Fig. 11. - Evolution du débit du déversoir (D), du nombre (N), et de la biomasse (B) d'organismes planctoniques exportés.

\section{4. - CYCLES BIOLOGIQUES}

La composition des populations de M. laciniatus, C. strenuus et D. longispina aux diverses dates de prélèvement, a été représentée sous forme d'histogrammes dans les figures 12 a, 12 b et 14 .

- Mixodiaptomus laciniatus (fig. 12 a).

Au dégel du lac, cette espèce est représentée par des adultes ô, et des $q$ ovigères et non ovigères. La ponte se poursuit jusqu’à la 
(3)

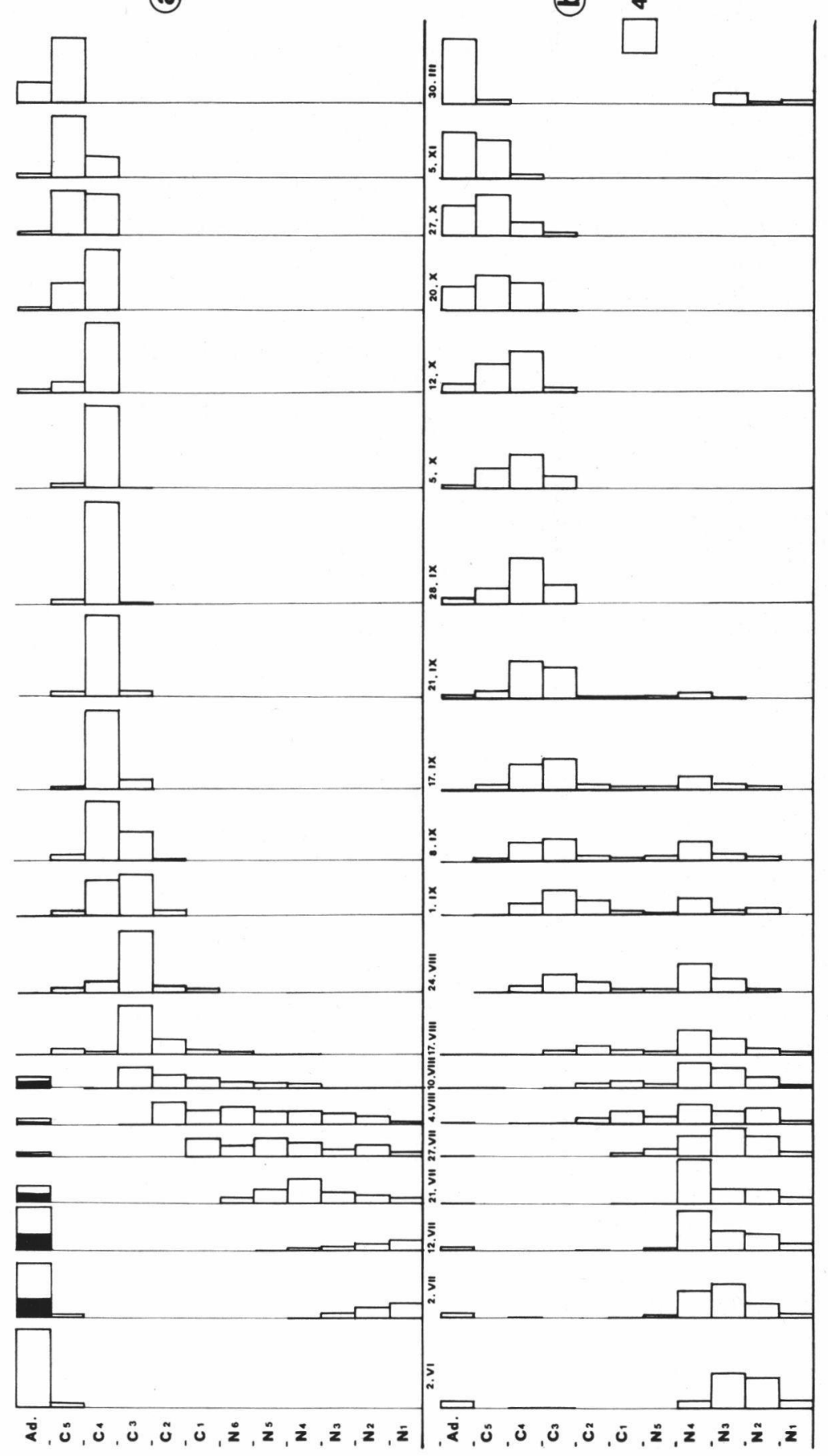

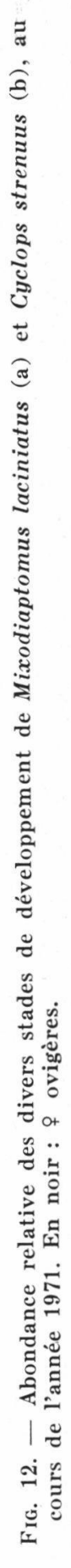


fin du mois de juillet tandis que le nombre d'individus sexués et le nombre moyen d'œufs portés par les $q$ ovigères diminuent progressivement. Les nauplii issus de cette phase de reproduction «printanière », atteignent leur densilé maximale à la fin du mois de juillet. Ils se transformeront au mois d'août en jeunes copépodites qui se différencieront à la fin du mois d'octobre en copépodites $\mathbf{V}$ o et o. Quelques individus atteignent le stade adulte à cette période de l'année où l'on retrouve un faible nombre de $q$ ovigères $(0,5$ à $1 \%$ de la population $)$.

La majeure partie des individus passera l'hiver au stade copépodite V, la fin de la croissance et le passage au stade adulte survenant entre les mois d'avril et mai, alors que le lac est encore gelé.

\section{- Cychops strenuus (fig. 12 b).}

Le cycle biologique de cette espèce a été établi par Rey et Dupin $(1973 \mathrm{~b})$, à partir de prélèvements de faune benthique réalisés au moyen d'une drague Petersen. La composition du peuplement planctonique permet de préciser et de compléter ce cycle qui se déroule selon un schéma semblable à celui indiqué pour $M$. laciniatus. Toutefois quelques variations sont à souligner :

a) La population est constituée au début de l'hiver par une forte proportion d'adultes (44\% de copépodites V, $52 \%$ d'adultes le 5-XI). En hiver, C. strenuus est représenté presque uniquement par des stades adultes (77\% le 20 -II, $96 \%$ le $30-\mathrm{III})$, ces adultes étant alors en période de reproduction. La phase de reproduction est en effet nettement plus précoce que celle de $M$. laciniatus. Le 30 -III, $15 \%$ des adultes sont des $q$ ovigères. Le 2-VI, on note $4 \%$ de $q$ ovigères et $83 \%$ de stades naupliens.

b) Les stades naupliens s'étalent largement dans le temps. La lecture des histogrammes révèlent $38 \%$ de stades N4, N5 fin août début septembre, alors que les adultes ont pratiquement disparu depuis la mi-juillet. Cet étalement des stades naupliens peut s'interpréter diversement :

- les adultes continuent à se reproduire mais, en raison de leur mode de vie benthique, n'apparaissent pas dans nos relevés. Cependant si l'on se réfère au cycle précédemment établi pour cette espèce (Rey et Dupin $1973 \mathrm{~b}$ ), on ne trouve également pas d'adultes dans les données benthiques dès la fin juillet;

- les stades naupliens de $C$. strenuus présentent un temps de développement plus long du fait des températures relativement basses régnant en profondeur.

Le taux de croissance des individus, assez faible au début de l'été, atteint sa valeur maximale à l'automne (fig. 13). 


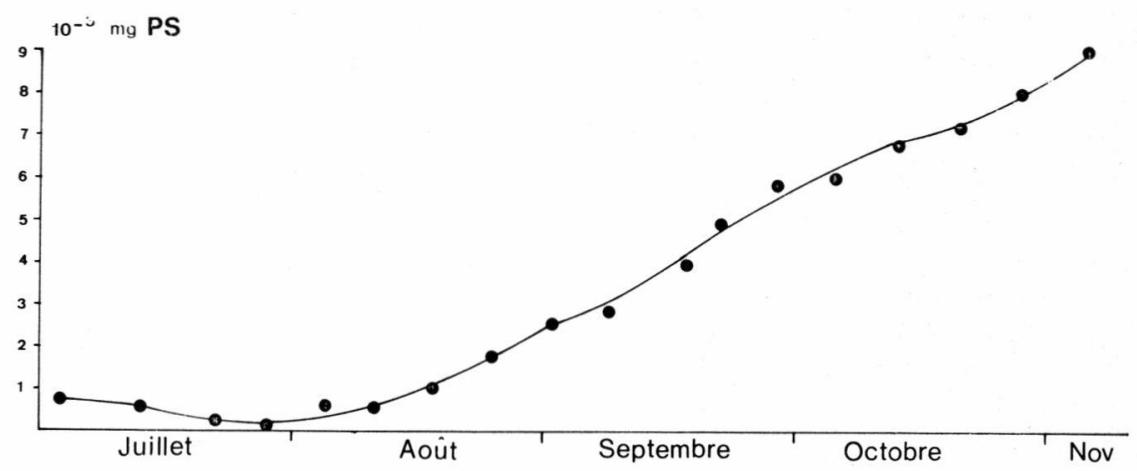

Fig. 13. - Evolution du poids moyen des individus planctoniques de Cyclops strenuus.

- Daphnia longispina (fig. 14).

Dans un travail antérieur (Rey 1968) nous avions décrit le cycle biologique de cette espèce au lac de Port-Bielh. La séparation des individus par classe de taille de $0,2 \mathrm{~mm}$, permet de préciser les résultats précédents.

L'éclosion des éphippies pendant le mois de juillet donne naissance à des $q$ dont la taille augmente rapidement. La maturité sexuelle est atteinte pour une longueur de 1,5 à $1,6 \mathrm{~mm}$, fin juillet début août.

Le développement des œufs pondus donne naissance à une deuxième génération de $q$ qui deviennent matures $(1,6 \mathrm{~mm})$ au début $\mathrm{du}$ mois de septembre. Ces $q$ de deuxième génération mettent donc environ deux fois plus de temps pour atteindre leur maturité (5 à 6 semaines contre 3 semaines en moyenne pour les $q$ issues des éphippies), et ceci malgré des conditions de température apparemment plus favorables. Outre leur vitesse de développement moindre, ces $q$ de deuxième génération montrent également un taux de reproduction inférieur aux précédentes $(1,4$ œufs en moyenne par o contre 6,1). Il est possible que taux de reproduction plus fort et développement plus rapide des $q$ issues des éphippies, soient dus en partie à la grande richesse en réserve nutritives des œufs de durée.

Les derniers œufs pondus donnent naissance à une population de ô qui atteint un effectif maximum fin août-début septembre, moment où débute la phase de reproduction sexuée. La proportion de ㅇ éphippiales augmente alors progressivement jusqu’au gel.

\section{- ROTIFÈRES.}

Le nombre d'individus et la proportion de o ovigères de Keratella hiemalis diminuent progressivement depuis le dégel jusqu'à 


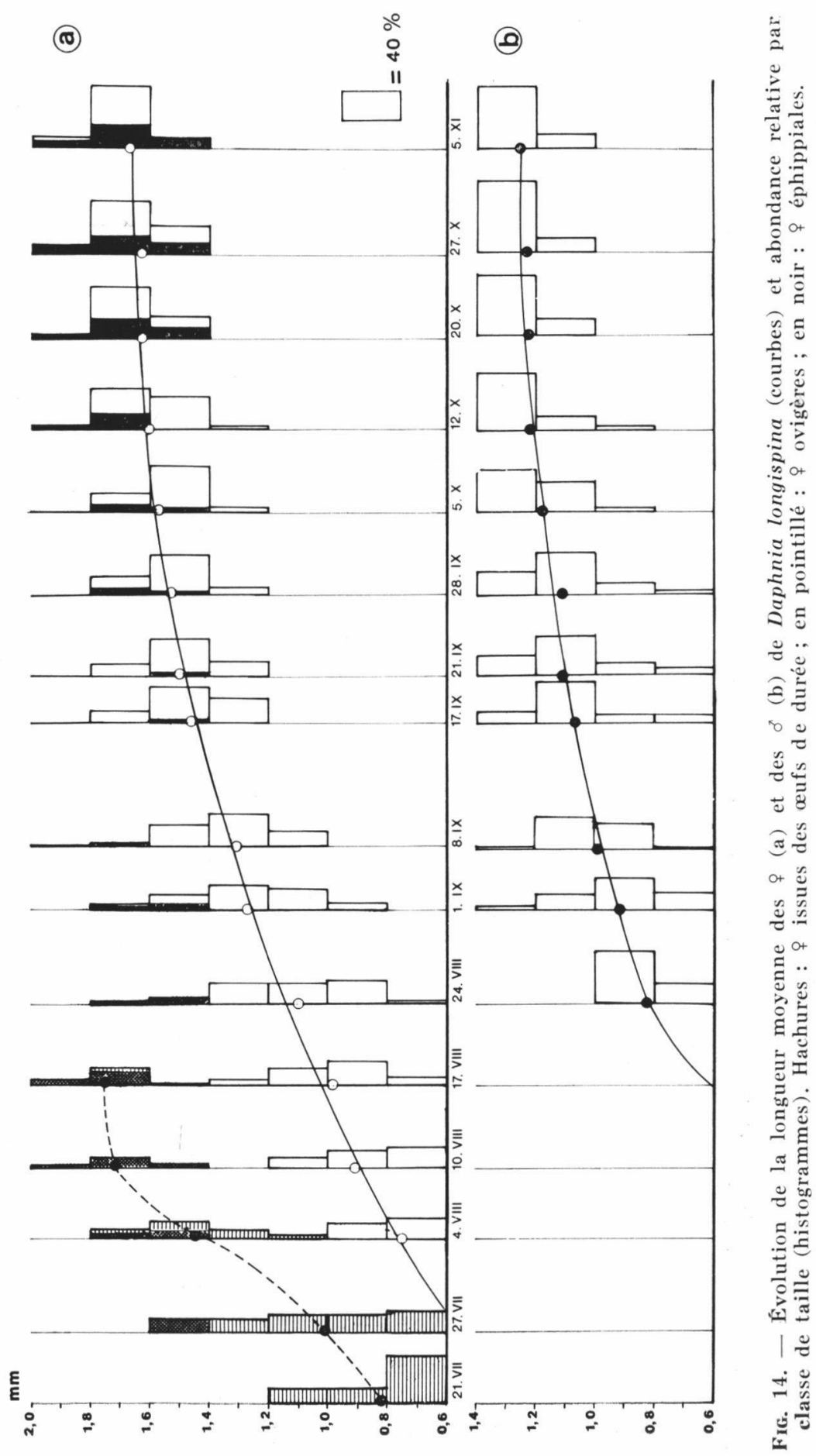


la fin du mois de juillet. Pour cette espèce sténotherme d'eau froide, la période de reproduction parthénogénétique maximale se situe vraisemblablement avant le dégel, à une température de $4{ }^{\circ} \mathrm{C}$. Le nombre d'œufs de durée augmente au cours du mois de juillet.

Les individus de Polyarthra vulgaris issus des oufs de durée, dépourvus d'ailettes (f. aptera-reducta), dominent dans les relevés de juillet. Les oufs amictiques donnent naissance à une seconde génération (f. typica), la première génération disparaissant le 15-VIII. Cette évolution est identique à celle que décrit Amren (1964) pour des populations de «ponds»du Spitzberg. La population s'accroît ensuite rapidement au mois d'août, pour atteindre un maximum en septembre. Le pourcentage de $q$ ovigères est élevé pendant toute la phase de croissance de la population : $100 \%$ à $73 \%$ entre le 21 -IX et le 10 -VIII. Il oscille ensuite pendant tout l'été entre 10 et $40 \%$.

Chez Ascomorpha ecaudis et Conochilus unicornis, la perte des œufs lors des prélèvement a rendu difficile l'établissement de leur cycle évolutif. La densité de $A$. ecaudis atteint un maximum le 10-VIII, celle de $C$. unicornis se situe entre le $1^{\text {er }}$ et le 8 septembre.

\section{5. - BIOMASSE ET PRODUCTION}

\section{1. - Paramètres de base et méthodes d'évaluation.}

L'échantillonnage quantitatif fournit une image des populations aux diverses étapes de leur cycle biologique qui permet d'estimer la biomasse (B) si l'on connaît le poids des différents stades :

$$
\mathrm{B}=\mathbf{N}_{10} \cdot \mathrm{p}_{10}+\mathbf{N}_{1} \cdot \mathbf{p}_{1}+\ldots+\mathbf{N}_{\mathrm{i}} \cdot \mathrm{p}_{\mathrm{i}},
$$

où $\mathrm{N}_{0}, \mathrm{~N}_{1}, \mathrm{~N}_{\mathrm{i}}$ et $\mathrm{p}_{0}, \mathrm{p}_{1}, \mathrm{p}_{\mathrm{i}}$ sont respectivement les effectifs et les poids d'un individu aux différents stades, depuis l'œuf jusqu'à l'adulte; $\mathrm{p}_{0}, \mathrm{p}_{1}, \mathrm{p}_{\mathrm{i}}$ sont déterminés à partir des relations taille poids (fig. 1).

Les fluctuations de la biomasse résultent à la fois de l'accroissement des nombres et du poids des individus, et de l'élimination par mortalité.

Les diverses méthodes d'évaluation de la production $(\mathrm{P})$ revues par Winberg (1971) et par Hillbricht-Ilkowska et Patalas (1967), sont fondées sur l'estimation de l'un des deux paramètres suivants :

1) augmentation pondérale des individus entre 2 relevés :

$$
\mathrm{P}=\left(\mathrm{p}_{2}-\mathrm{p}_{1}\right) \cdot\left(\frac{\mathrm{N}_{2}-\mathrm{N}_{1}}{2}\right)
$$

2 ) nombre d'individus éléminés $\left(\mathrm{N}_{\mathrm{e}}\right)$ entre 2 relevés :

$$
\mathrm{P}=\mathrm{N}_{\mathrm{e}} \cdot \overline{\mathrm{p}}+\left(\mathrm{N}_{2} \mathrm{p}_{2}-\mathrm{N}_{1} \mathrm{p}_{1}\right) \text {. }
$$




\subsection{1. - Evolution DES NOMBRES.}

Les courbes de diminution des nombres, généralement employées pour calculer la production de poissons (Allen 1951) ou d'invertébrés benthiques (Ness et Dugdale 1959, Laville 1972 b, Décamps et Lafont 1974), sont également utilisables dans le cas de populations planctoniques à courte période de reproduction suivie d'un déclin de la population. L'évolution numérique de la population de Mixodiaptomus laciniatus et, lorsqu'on les considère séparément, des populations de \& et de 우 de $D$. longispina, permet d'estimer la mortalité de la population dès la fin de la reproduction (fig. 12 a et 14).

Durant la phase de reproduction, élimina!ion et recrutement ont lieu simultanément. La mortalité peut alors être estimée par différence entre le taux de natalité et le taux de variation réel de la population. Cette méthode utilisée par Elster (1954), Elster et Schwoerbel (1970), Wright (1965) pour les Crustacés, et par Edmonson $(1960,1965)$ pour les Rolifères, est baséc sur l'estimation du taux de reproduction $\mathrm{B}^{\prime}$ :

$$
\mathrm{B}^{\prime}=\frac{\mathrm{E}}{\mathrm{D}}=\frac{\text { nombre d'œufs } / q}{\text { durée de développement de l'œuf }}
$$

a) En l'absence de mortalité et dans le cas où l'intervalle de temps ( $t$ ) entre échantillons successifs n'excède pas $D$, on peut admettre une croissance linéaire des nombres. Au bout du temps $t$, la population devrait atteindre un effectif théorique $\left(N^{\prime}{ }_{t}\right)$ tel que

$$
\mathbf{N}^{\prime}{ }_{t}=B^{\prime} \cdot t \cdot N_{0} .
$$

Le nombre d'individus éliminés sera :

$$
\mathrm{N}_{\mathrm{e}}=\mathrm{N}_{\mathrm{t}}^{\prime}-\mathrm{N}^{\prime}=\text { effectif théorique - effectif observé. }
$$

b) Lorsque $t$ est supérieur à $\mathrm{D}$ (Rotifères), on peut admettre une croissance exponentielle de la population. En l'absence de mortalité, on aurait alors :

$$
\mathbf{N}^{\prime}{ }_{t}=\mathbf{N}_{00} \cdot \mathrm{e}^{b^{\prime \prime} t}
$$

où $b^{\prime}=$ taux instantané de natalité $=L_{n}\left(B^{\prime}+1\right)$.

L'évolution réelle de la population pouvant s'exprimer par :

$$
N_{t}=N_{o} \cdot e^{r^{\prime} t}=N_{0} \cdot e^{\left(b^{\prime}-d^{\prime \prime}\right) t}
$$

où $\mathbf{r}^{\prime}=$ taux instantané de croissance de la population

$\mathrm{d}^{\prime}=$ taux instantané de mortalité,

on peut estimer la proportion d'individus éliminés quotidiennement

$$
\mathbf{M}=\left(\mathrm{B}^{\prime}+1\right)-\mathrm{e}^{\mathrm{r}^{\prime}} .
$$




\subsection{2. - Croissance PONisérale des individus.}

Elle a été évaluéc par deux méthodes, à partir des relations taille - poids.

a) Connaissant la proportion des divers stades de développement (Copépodes) ou la distribution des tailles (Cladocères) dans la population, on peut calculer le poids moyen $(\bar{p})$ d'un individu lors de chaque relevé. L'augmentation journalière du poids entre 2 relevés est donné par la formule :

$$
\Delta \bar{p}=\frac{\bar{p}_{2}-\bar{p}_{1}}{t_{2}-t_{1}}
$$

b) Connaissant les poids initial ef final d'un stade $\mathbf{i}$ et sa durée de développement $\left(D_{1}\right)$, l'augmentation journalière du poids de ce stade est donnée par la formule :

$$
\Delta p_{i}=\frac{p_{\text {finaI }}-p_{\text {intial }}}{D_{i}}
$$

Les poids initiaux et finaux des divers stades ont été déterminés à partir de la courbe d'évolution du poids des individus en fonction de leur âge (fig. 19 et 24 a et b).

L'examen des prélèvements donne rarement la possibilité d'évaluer la durée de développement des divers stades. Lo temps écoulé entre les premières apparitions d'individus dans deux stades successifs, ou la distance en're les pics des courbes des nombres, fournissent des valeurs dont la précision est fonction de la fréquence d'échantillonnage. Exception faite pour les oeufs, les résultats d'élevage sont par ailleurs difficilement transposables au milieu naturel dont ils ne reproduisent souvent que les conditions thermiques. L'évolution des populations de Crustacés du lac de PortBielh permet d'estimer le temps de développement moyen des divers stades. Le calcul repose sur le raisonnement suivant:

Soient $N_{1}$ le nombre d'individus d'un stade i présents dans le lac à l'instant $\mathrm{t}$, et $\mathrm{D}_{\mathrm{i}}$ la durée de ce stade.

Le nombre d'individus passant all stade suivant en 1 jour sera égal à : $\frac{N_{i}}{D_{i}}$.

Pendant les $\mathrm{T}$ jours que dure la présence de ce stade dans le milieu, le nombre d'individus passant au stade suivant sera égal à :

$$
\int_{0}^{T} \frac{N_{1}}{D_{i}}
$$

ou encore à $N$ : nombre moyen d'individus présents dans le milieu 
pendant la période $\mathrm{T}$, puisque tous les individus de la population passent par le stade $\mathrm{i}$.

$\mathrm{N}$ pouvant être estimé à partir de la courbe de mortalité, la valeur moyenne de $\mathrm{D}_{\mathrm{i}}$ pendant la période $(\mathrm{T})$ d'existence du stade $\mathrm{i}$ sera donnée par :

$$
\overline{\mathrm{D}}_{\mathrm{i}}=\frac{1}{\mathrm{~N}} \int_{0}^{\mathrm{T}} \mathrm{N}_{\mathrm{i}}
$$

Ce raisonnement est illustré par le schéma de la figure 15 qui représente l'évolution d'un population théorique de 10 individus (numérotés de a à j) passant par trois stades successifs d'une durée de 1,2 et 3 jours, avec mort d'un individu entre chaque stade.
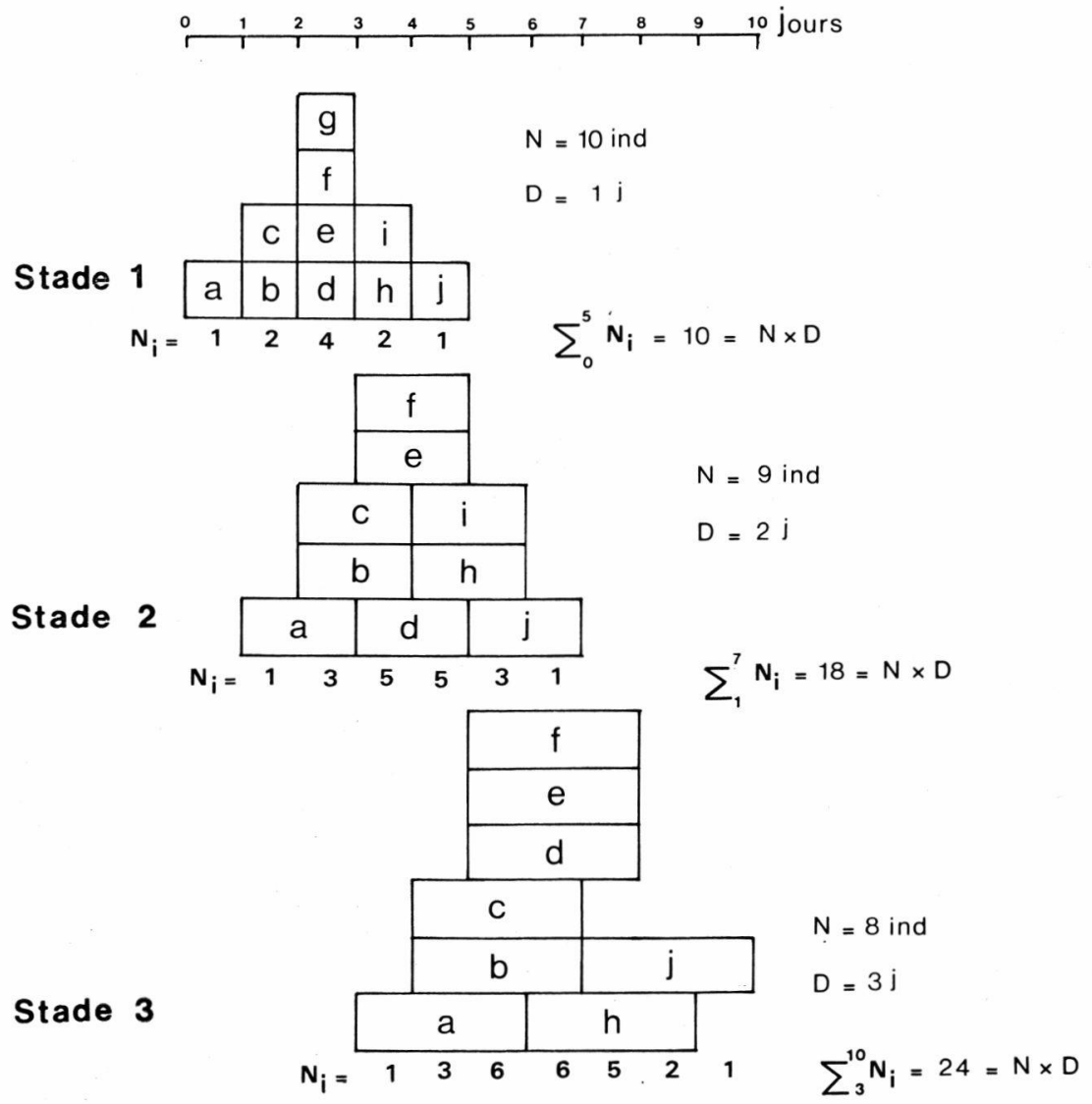

Fig. 15. - Evolution d'une population théorique passant par 3 stades successifs d'une durée (D) de $1,2,3$ jours avec mort d'1 individu entre chaque stade. 
Dans un but de comparaison, nous avons utilisé ces diverses méthodes pour calculer la production secondaire des trois espèces dominantes du zooplancton du Iac de Port-Bielh : Mixodiaptomus laciniatus, Daphnia longispina, Polyarthra vulgaris. Les résultats, exprimés par $\mathrm{m}^{3}$ d'eau, représentent la moyenne de la population présente dans une colonne d'eau de 18 mètres de profondeur et de $1 \mathrm{~m}^{2}$ de section.

Ils seront ensuite corrigés en tenant compte de la distribution verticale des organismes et des paramètres morphométriques du lac, afin d'obtenir une évaluation de la production secondaire par $\mathrm{m}^{2}$ moyen de lac.

\section{2. - Mixodiaptomus laciniatus.}

5.2.1. - Évolution dES NOMBRES ET dURÉE DE DÉvELOPPEMENT.

La densité des individus appartenant aux divers stades de développement de cette espèce et son évolution dans le temps apparaissent dans les figures 16 et 17 .

La reproduction débute avant même la fusion complète de la glace et l'on peut estimer que près de $80 \%$ des œufs ont été pondus avant le 21 juillet, en 1971. A cette époque, la population atteint sa densité optimale avec 30 ind./litre d'eau ; $80 \%$ de la population sont représentés par des stades naupliens issus de ces pontes.

Dès la fin de la période de reproduction (10 août), la population décroît progressivement et l'évolution des nombres peut être décrite par l'équation :

$$
\mathbf{N}_{\mathrm{t}}=\mathbf{N}_{\mathrm{o}} \cdot \mathrm{e}^{-0,00537 \mathrm{t}}
$$

Le taux journalier de mortalité $M$ est donc de $0,53 \%$ de la population. Nous avons vu $(\S 3.3 . \mathrm{b})$, que la dérive des organismes au déversoir représentait environ la moitié de cette morlalité au début du mois d'août, le quart au début d'octobre.

L'estimation de l'effectif de la population à partir de l'équation précédente, et les courbes d'évolution des nombres des différents stades de développement (fig. 16), ont été utilisés pour calculer la durée moyenne $\left(\widehat{D}_{1}\right)$ de développement de chaque stade.

Les résultats sont reportés dans le tableau III, qui donne également la température moyenne du milieu pendant la période où le stade considéré est présent.

Ces résultats sont tout à fait comparables à ceux de Eichorn (1957) qui a déterminé expérimentalement la durée de développement de l'œuf $\left(14,9 \mathrm{j}\right.$ à $5^{\circ} ; 12,7 \mathrm{j}$ à $\left.6^{\circ}\right)$, et de l'orthonauplius $\left(2,3 \mathrm{j}\right.$ à $6^{\circ} ; 2,0 \mathrm{j}$ à $\left.7^{\circ}\right)$ de $M$. laciniatus. 


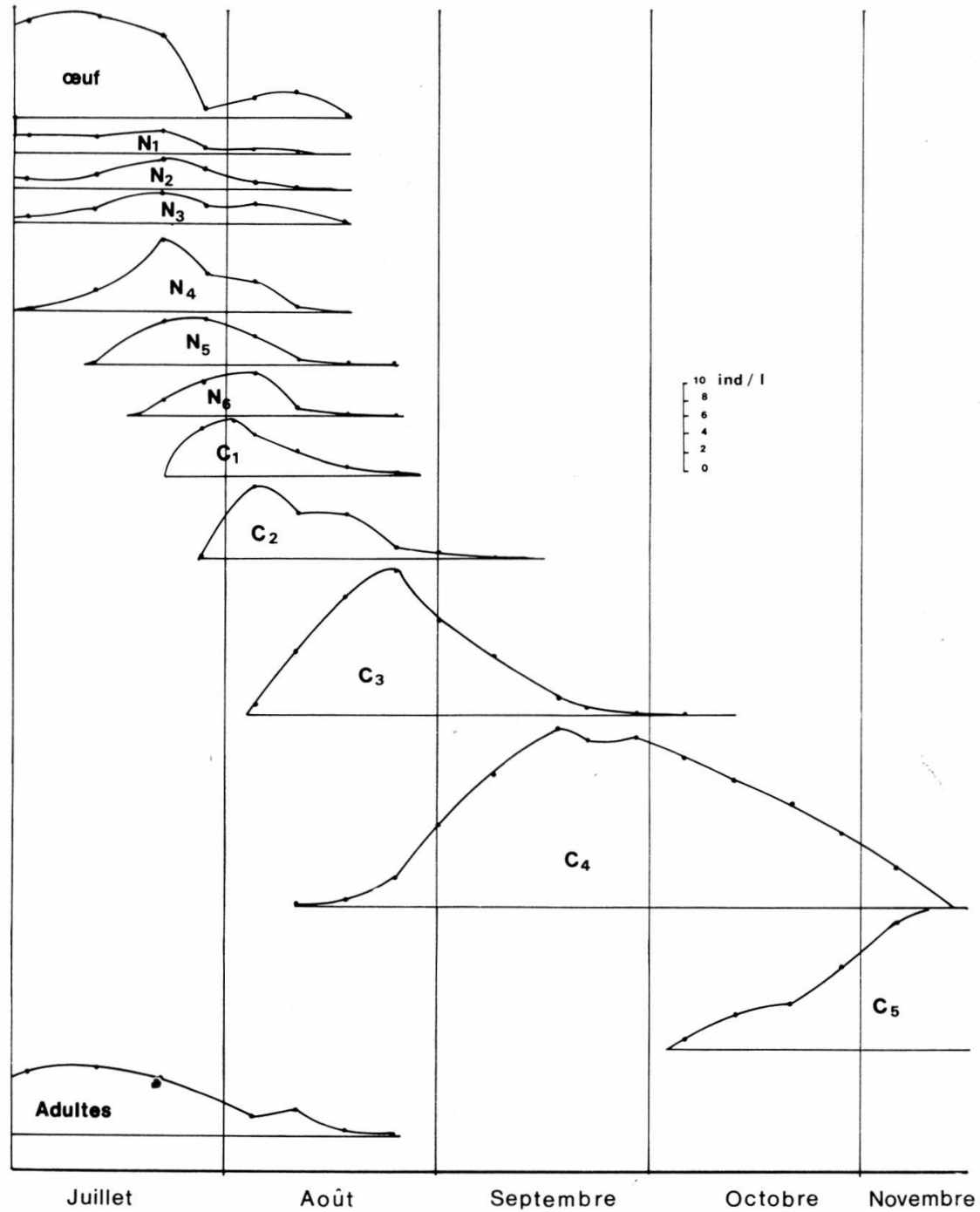

Fig. 16. - Evolution du nombre moyen d'individus de Mixodiaptomus laciniatus aux différents stades de développement.

De même, l'œuf de Eudiaptomus gracilis se développe en 17,9 j à $5^{\circ}$ et $14,4 \mathrm{j}$ à $7^{\circ}$ (Elster, 1954). Eckstein (1963) estime que le développement de l'œuf de Eudiaptomus graciloüdes dure 13,1 j à $5^{\circ}$, celui des stades naupliens $20 \mathrm{j}\left(10^{\circ}\right)$ à $26 \mathrm{j}\left(8^{\circ}\right)$, celui des stades copépodites $34 \mathrm{j}\left(10^{\circ}\right)$ à $41 \mathrm{j}\left(8^{\circ}\right)$. 


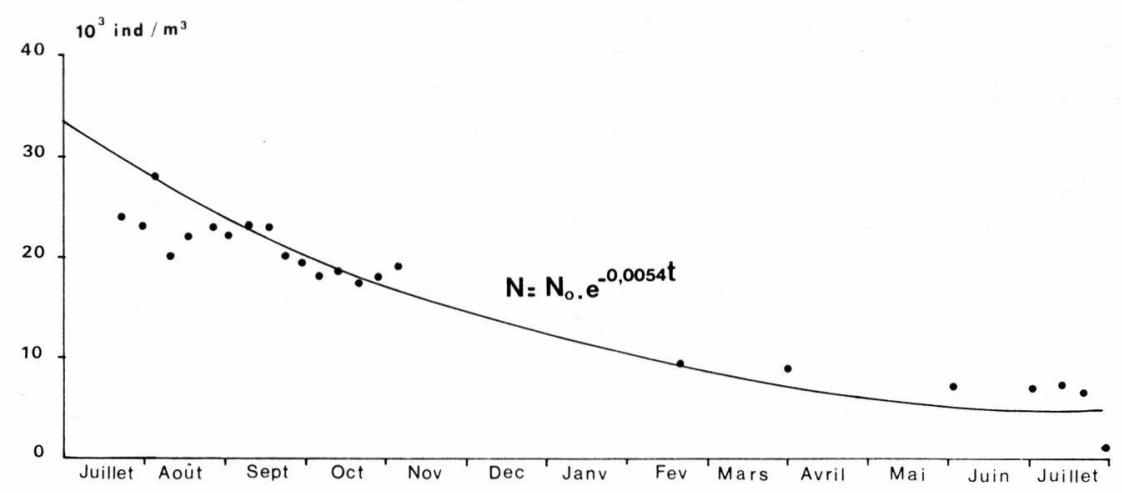

FIg. 17. - Courbe de mortalité de Mixodiaptomus laciniatus.

TableaU III. - Durée moyenne $\left(\overline{\mathrm{D}_{\mathrm{i}}}\right)$ des différents stades de Mixodiaptomus laciniatus. La température moyenne du milieu pendant la durée de développement du stade, a été calculée en tenant compte de la répartition verticale des organismes (cf. fig. 3).

\begin{tabular}{ccc}
\hline & $\overline{D_{i}}$ & $\mathrm{~T}^{\circ} \mathrm{C}$ \\
Stade & $(\mathrm{j})$ & \\
\hline & 14 & $5^{\circ} 4$ \\
$\mathrm{~N}_{1}$ & 2,3 & $6^{\circ} 7$ \\
$\mathrm{~N}_{2}$ & 2,3 & $6^{\circ} 7$ \\
$\mathrm{~N}_{3}$ & 2,5 & $8^{\circ} 7$ \\
$\mathrm{~N}_{4}$ & 4,0 & $8^{\circ} 7$ \\
$\mathrm{~N}_{5}$ & 3,5 & $9^{\circ} 3$ \\
$\mathrm{~N}_{6}$ & 3,0 & $10^{\circ} 2$ \\
$\mathrm{C}_{1}$ & 3,5 & $10^{\circ} 3$ \\
$\mathrm{C}_{2}$ & 4,5 & $10^{\circ} 7$ \\
$\mathrm{C}_{3}$ & 19,0 & $10^{\circ} 3$ \\
$\mathrm{C}_{4}$ & 52,5 & $9^{\circ} 8$ \\
$\mathrm{C}_{5}$ & 237,0 & $4^{\circ}$ \\
\hline
\end{tabular}

Selon Sushkina (1964), le temps de développement des Diaptomides à une $\mathrm{T}^{\circ}$ de $10^{\circ}$ et une concentration de nourriture comprise entre 0,2 et $1 \mathrm{~g} / \mathrm{m}^{2}$, est de $27 \mathrm{j}$ pour les nauplii et de $31 \mathrm{j}$ pour les copépodites.

\subsection{2. - Croissance pondérale.}

a) Le poids d'un individu de chaque stade et la composition de la population à chaque relevé, permettent le calcul de la biomasse (B) et du poids moyen d'un individu.

Par exemple, le 5.XI, la population (19 $550 \mathrm{ind} / \mathrm{m}^{3}$ ) est composée de 4650 copépodites IV $(3,5 \mu \mathrm{g}), 14500$ copépodites $\mathrm{V}(5,0 \mu), 100$ 命 $(8 \mu \mathrm{g}), 100$ ㅇ $(10,6 \mu \mathrm{g})$ et 100 o ovigères $(11,1 \mu \mathrm{g})$. 
La biomasse $\mathrm{B}=(4650 \times 3,5)+(14500 \times 5)+(100 \times 8)$ $+(100 \times 10,6)+(100 \times 11,1)=89,072 \mathrm{mg}$, et le poids moyen d'un individu $\overline{\mathrm{p}}=\frac{89,072}{19550}=4,55 \mu \mathrm{g}$.

L'évolution du poids moyen des individus au cours de l'été a été représentée dans la figure 18 .

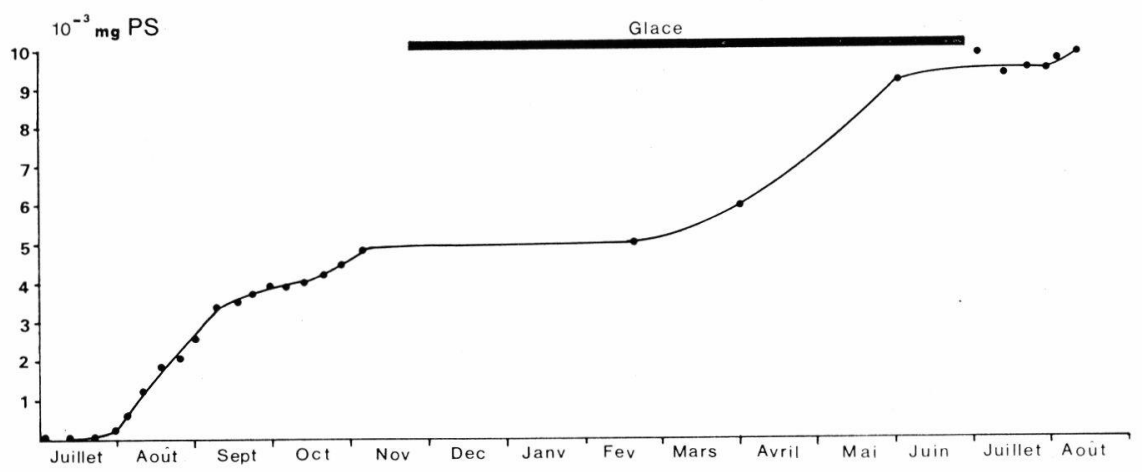

Fig. 18. - Evolution du poids moyen d'un individu de Mixodiaptomus laciniatus.

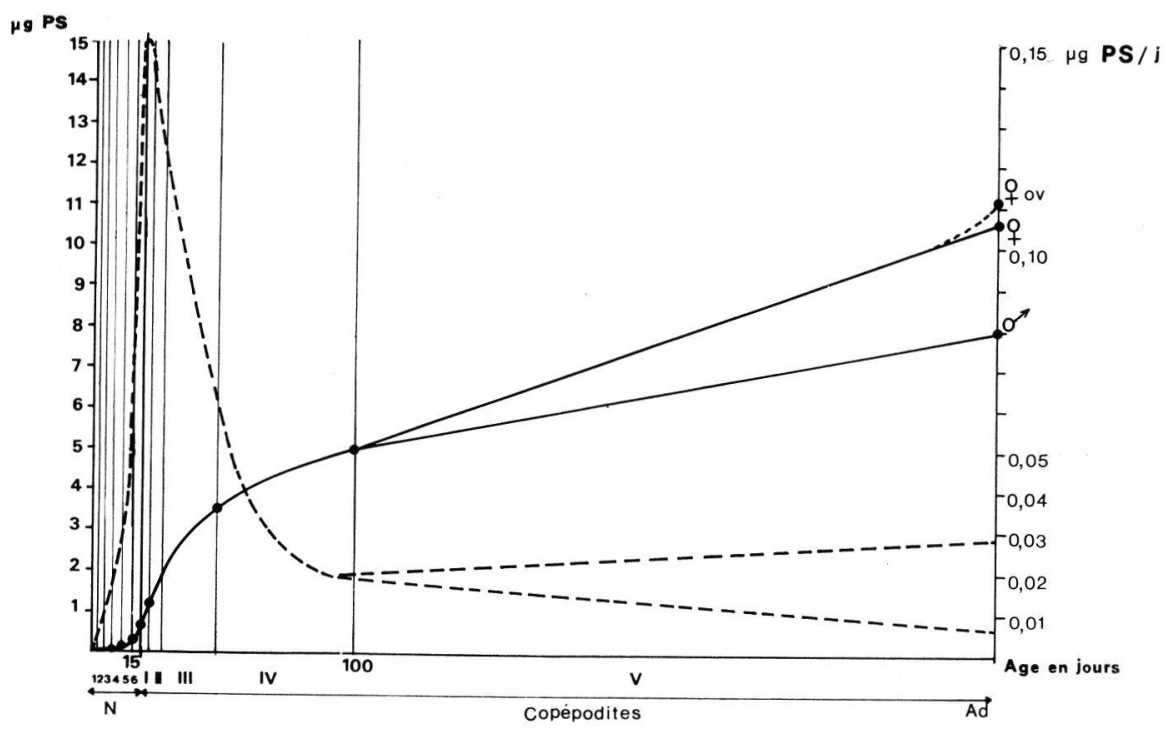

Fig. 19. - Variation du poids individuel (trait plein) et augmentation journalière du poids (tirets) en fonction de l'âge, chez Mixodiaptomus laciniatus. 
Pour calculer $\bar{p}$ au mois de juillet, nous avons distingué les ndividus de l'année (stades naupliens), des individus de la cohorte précédente (adultes) dont nous avons reporté le poids en fin de courbe.

La courbe de croissance de la figure 18 indique une augmentation du poids plus rapide en août, période de température optimale de l'eau. Le ralentissement de la croissance à partir du mois de septembre peut être attribué à une diminution de la température de l'eau, ou traduire l'existence d'une concurrence alimentaire entre organismes. Durant l'automne en effet, la biomasse de phytoplancton est maintenue à un niveau relativement faible, du fait d'une intense exploitation par le zooplancton (Capblancq 1972).

L'augmentation du poids est nulle pendant la plus grande partie de l'hiver, mais il est à noter que la croissance des individus repart sous la glace an mois d'avril.

b) Les courbes de la figure 19 ont été tracées en admettant que le poids d'un stade donné constitue le poids initial du stade suivant (courbe a). Par différenciation, cette courbe donne l'accroissement journalier du poids en fonction de l'âge (courbe b).

Le taux de croissance optimal correspond au passage du stade uauplius 6 au stade copépodite III. L’augmentation journalière du poids se ralentit aux stades copépodites IV et $\mathrm{V}$, par suite de l'allongement de la durée de développement.

\subsection{3. - BIOMASSE ET PRODUCTION DE Mixodiaptomus laciniatus.}

Elles ont été calculées à partir des paramères précédents, par deux méthodes différentes et leur variante graphique :

1 a - Accroissement du poids moyen d'un individu, multiplié par le nombre moyen d'individus présents entre deux échantillons successifs.

$1 \mathrm{~b}$ - Courbe de croissance-survie, ou méthode graphique d'Allen.

$2 a$ - Accroissement journalier moyen des divers stades, multiplié par l'effectif de chaque stade.

$2 \mathrm{~b}$ - Méthode graphique de Winberg, Pecen el Sushkina (1965).

Les résultats sont regroupés dans le tableau IV et les figures 20 et 21. Minimale à la fin du mois de juillet (période de disparition des adultes et population composéc essentiellement de stades naupliens), la biomasse augmente rapidement au mois d'août pour atteindre, dès le mois de septembre, sa valeur optimale. Elle se maintient ensuite à une valeur à peu près constante jusqu'au gel.

La courbe de croissance-survic (fig. 20) présente trois points d'inflexion qui marquent le ralentissement (mi-septembre), l'arrêt (novembre à février) et la reprise (mars) de la croissance. La production hivernale (novembre à juin) qui correspond au passage des 
Tableau IV. - Estimation de la biomasse et de la production moyennes de Mixodiaptomus laciniatus, d'après l'évolution du poids moyen des individus.

\begin{tabular}{|c|c|c|c|c|c|c|c|c|c|c|}
\hline \multirow[b]{2}{*}{ Date } & \multicolumn{4}{|c|}{ Population 1970} & \multicolumn{3}{|c|}{ Population 1971} & \multirow[b]{2}{*}{$\begin{array}{c}\mathbf{P} \\
\left(\mathrm{mg} / \mathrm{m}^{3} / \mathrm{j}\right) \\
\mathrm{PS}\end{array}$} & \multirow[b]{2}{*}{$\begin{array}{c}\mathrm{B} \\
\left(\mathrm{mg} / \mathrm{m}^{3}\right) \\
\mathrm{PS}\end{array}$} & \multirow[b]{2}{*}{$\begin{array}{c}\mathrm{P} / \mathrm{B} \\
\times 100\end{array}$} \\
\hline & $\Delta t$ & $\begin{array}{c}\text { Poids } \\
\text { moy. } \\
(\mu \mathrm{g} \mathrm{PS})\end{array}$ & $\begin{array}{c}\Delta \mathrm{P} / \mathrm{j} \\
(\mu \mathrm{g} \mathrm{PS})\end{array}$ & $\mathrm{N}$ & $\begin{array}{c}\text { Poids } \\
\text { moy. } \\
(\mu \mathrm{g} \mathrm{PS})\end{array}$ & $\begin{array}{c}\Delta \mathrm{P} / \mathrm{j} \\
(\mu \mathrm{g} P S)\end{array}$ & $\mathrm{N}$ & & & \\
\hline 2-VII & & 9,81 & & 7090 & 0,02 & & 3870 & & 69,61 & \\
\hline 12-ViII & 10 & 9,43 & - & 7518 & 0,03 & 0,002 & 6685 & 0,009 & 71,16 & 0,01 \\
\hline 21-VII & 9 & 9,48 & 0,05 & 6533 & 0,08 & 0,005 & 24130 & 0,11 & 63,78 & 0,17 \\
\hline 27-VII & 6 & 9,53 & 0,05 & 845 & 0,23 & 0,026 & 23023 & 0,63 & 13,33 & 1,64 \\
\hline 4-VIII & 8 & 9,49 & - & 1993 & 0,61 & 0,048 & 28189 & 1,22 & 36,15 & 4,95 \\
\hline 10-ViIII & 6 & 9,70 & 0,21 & 3008 & 1,21 & 0,100 & 16460 & 2,32 & 49,13 & 5,45 \\
\hline 17-VIII & 7 & 9,93 & $\begin{array}{l}0,23 \\
0,70\end{array}$ & 477 & 1,65 & $\begin{array}{l}0,003 \\
0,086\end{array}$ & 22683 & 1,98 & 46,81 & 4,19 \\
\hline 24-VIII & 8 & 10,63 & & & $\begin{array}{l}2,25 \\
, 70\end{array}$ & 0,069 & 22852 & 1,54 & $\begin{array}{l}47,77 \\
5768\end{array}$ & 2,93 \\
\hline $\begin{array}{l}\text { 1-IX } \\
\text { 8-IX }\end{array}$ & 7 & & & & $\begin{array}{l}2,70 \\
3,30\end{array}$ & 0,086 & $\begin{array}{r}22050 \\
23259\end{array}$ & 1,94 & $\begin{array}{l}57,68 \\
80,27\end{array}$ & 2,82 \\
\hline 17-IX & 9 & & & & 3.53 & 0,026 & 22713 & 0,59 & 80,30 & 0,74 \\
\hline 21-IX & 4 & & & & 3,73 & 0,05 & 20022 & 1,07 & 74,67 & 1,38 \\
\hline 28-IX & 7 & & & & 3,90 & 0,018 & 19783 & 0,33 & 77,99 & 0,44 \\
\hline $5-\mathrm{X}$ & 7 & & & & 3,92 & 0,02 & 18296 & 0,37 & 71,79 & 0,50 \\
\hline $12-X$ & 8 & & & & 4,06 & 0,025 & 18639 & 0,45 & 75,71 & 0,60 \\
\hline 20-X & & & & & 4,26 & & 17310 & & 73,82 & \\
\hline 27-X & 7 & & & & 4,52 & 0,052 & 18220 & 0,91 & 82,33 & 1,17 \\
\hline 5-XI & 9 & & & & 4,84 & 0,036 & 19189 & 0,66 & 92,37 & 0,77 \\
\hline
\end{tabular}




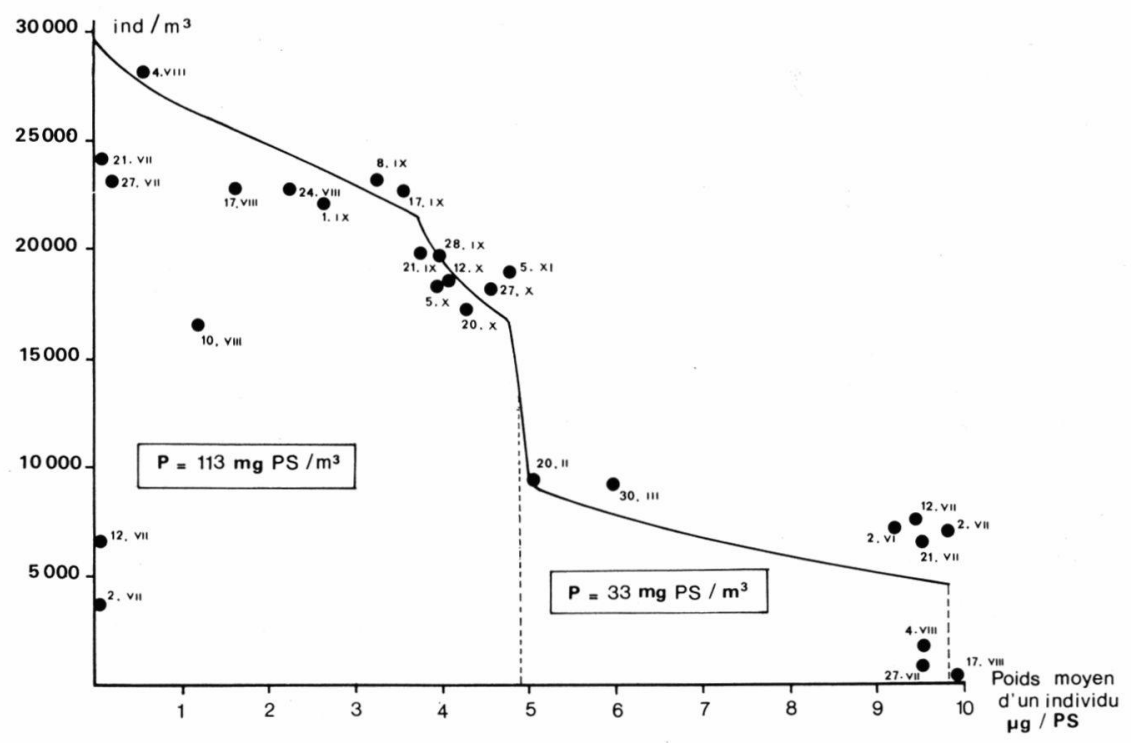

Fig. 20. - Courbe croissance-survie de Mixodiaptomus laciniatus : la courbe représente l'évolution des nombres estimée d'après l'équation de mortalité. La production annuelle moyenne, calculée par intégration des courbes, est indiquée sur la figure.

stades copépodites $\mathrm{V}$ aux adultes matures, équivaut à $23 \%$ de la production annuelle de $M$. laciniatus.

Les valeurs de la production diffèrent légèrement selon la méthode de calcul utilisée. La représentation graphique des résultats (fig. 21) révèle cependant que, quelle que soit la méthode d'évaluation, la production présente un maximum au mois d'août, cette période représentant près de $50 \%$ de la production annuelle de M. laciniatus. En automne, les taux de production journaliers sont 3 à 6 fois plus faibles et relativement constants jusqu'au gel.

Le coefficient $\mathrm{P} / \mathrm{B}$ ou taux d'accroissement journalier de la biomasse, présente un pic au début du mois d'août (fig. 21) et décroît ensuite progressivement jusqu'à l'automne. Le rapport de la production annuelle à la biomasse moyenne de la population $(\mathrm{P} / \overline{\mathrm{B}})$, varie entre 2,25 et 2,75 ; calculé par rapport à la biomasse maximale du 5.XI, il varie de 1,35 à 1,65 selon la méthode utilisée.

Ces dernières valeurs sont comparables à celles qu'indiquent Laville (1971 b), Giani et Laville (1973), Décamps et Lafont (1974) pour des larves d'insectes aquatiques d'altitude. 


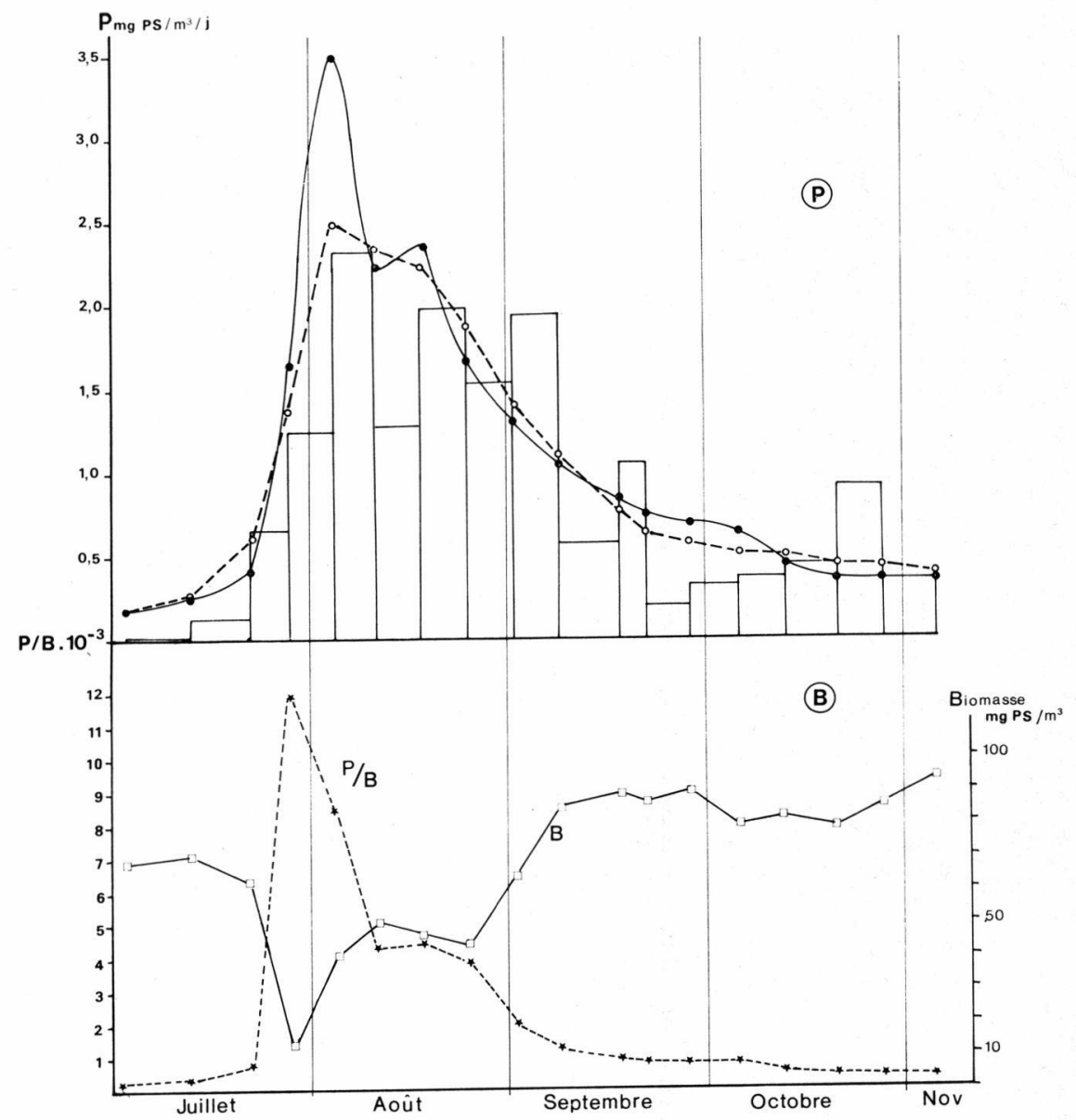

FIg. 21. - Evolution de la production moyenne (P), de la biomasse moyenne (B) et du rapport $\mathrm{P} / \mathrm{B}$ chez Mixodiaptomus laciniatus. Les résultats de la production en $\mathrm{mg}$ P.S./ $\mathrm{m}^{3} / \mathrm{j}$ correspondent à la méthode 1 a (histogrammes), 2 a (tirets - cercles blancs) et $2 \mathrm{~b}$ (trait plein - cercles noirs).

\section{3. - Daphnia longispina.}

\subsection{1. — Évolution des NOMBRES ET dURÉE DE dÉvelopPEMENT.}

Comparativement à la durée de vie, la période de reproduction de Daphnia longispina est relativement plus longue que celle de $M$. laciniatus. L'étude des cycles biologiques a montré cependant que les $\hat{o}$ apparaissent 1 mois après les $q$ ovigères et proviennent, en toute vraisemblance, des derniers oufs pondus.

Si on considère séparément l'évolution du nombre des $q$ issues des œufs pondus fin juillet début août, et du nombre des ô issus des œufs pondus fin août-début septembre, chaque population 
Tableau V. - Dynamique de population et production de Daphnia longispina. Le temps de développement des aufs se réfère aux données Elster et Schwoerbel (1970).

\begin{tabular}{|c|c|c|c|c|c|c|c|c|c|c|c|c|c|c|}
\hline Date & $\Delta \mathrm{t}$ & $\mathrm{N}$ & $\omega$ & $\mathrm{T}^{\circ} \mathrm{C}$ & $1 / D$ & $\bar{\omega}$ & $\begin{array}{l}\text { Accrois- } \\
\text { sement } \\
\text { théori- } \\
\text { que/j } \\
-1 / D \\
(1)\end{array}$ & $\begin{array}{c}\text { Accrois- } \\
\text { sement } \\
\text { réel } \\
\mathrm{N}_{2}-\mathrm{N}_{1} \\
\Delta \mathrm{t} \\
(2)\end{array}$ & $\begin{array}{l}\text { Pertes/j } \\
(1)-(2)\end{array}$ & $\begin{array}{c}\text { Pertes } \\
\%\end{array}$ & $\begin{array}{l}\text { N théo- } \\
\text { rique } \\
\text { sans } \\
\text { pertes }\end{array}$ & $\begin{array}{c}\text { N éli- } \\
\text { minés } \\
\text { entre } \\
2 \\
\text { relevés }\end{array}$ & $\begin{array}{l}\text { Poids } \\
\text { d'un } \\
\text { individu } \\
(\mu \mathrm{g} \text { PS })\end{array}$ & $\begin{array}{c}\text { Produc- } \\
\text { tion } \\
\text { journa- } \\
\text { lière } \\
\text { (mgPS/j) }\end{array}$ \\
\hline 21-VII & & 248 & - & $6^{\circ} 75$ & & & & & & & 248 & & 1,56 & \\
\hline 27-ViII & 6 & 312 & 275 & $7^{\circ} 9$ & 0,085 & 137 & 12 & 11 & 1,0 & 0,35 & 318 & 6 & 3,60 & 0,13 \\
\hline 4-VIII & 8 & 385 & 605 & $9^{\circ} 1$ & 0,103 & 440 & 45 & 9 & 36 & 10,28 & & 287 & 5,35 & 0.28 \\
\hline 10-VIII & 6 & 1660 & 2850 & $9^{\circ} 8$ & 0,117 & 1727 & 201 & 212 & $(-11)$ & - & 1592 & - & 7,06 & 1,61 \\
\hline 17-VIII & 7 & 3025 & 3800 & $10^{\circ} 6$ & 0,130 & 3325 & 430 & 195 & 235 & 10,04 & 4672 & 1647 & 7,20 & 3,12 \\
\hline 24-VIII & 7 & 3610 & 630 & $11^{\circ} 4$ & 0,146 & 2215 & 322 & 84 & 238 & 7,18 & 5279 & 1669 & 4,12 & 1,35 \\
\hline 1-IX & $\begin{array}{l}8 \\
7\end{array}$ & 5555 & 590 & $11^{\circ} 7$ & 0,157 & 610 & $\begin{array}{r}95 \\
79\end{array}$ & 243 & $(-148)$ & - & 4372 & - & 6,72 & 2,81 \\
\hline 8-IX & 9 & 5631 & 385 & $12^{\circ}$ & 0,162 & 142 & 23 & $(-89)$ & -112 & 2,13 & 6109 & 1006 & 6,96 & 1,67 \\
\hline $\begin{array}{l}17-I X \\
21-I X\end{array}$ & 4 & $\begin{array}{l}4831 \\
4831\end{array}$ & $\begin{array}{r}150 \\
60\end{array}$ & $\begin{array}{l}11^{\circ} 6 \\
11^{\circ} 5\end{array}$ & 0,155 & 80 & 12 & $(-48)$ & -61 & 1,28 & $\begin{array}{l}5837 \\
4880\end{array}$ & 244 & $\begin{array}{l}9,50 \\
9,30\end{array}$ & 0,58 \\
\hline 28-IX & 7 & 5173 & 55 & $10^{\circ} 9$ & 0,154 & 57 & 9 & 77 & $(-68)$ & - & 4698 & - & 10,22 & 1,08 \\
\hline 5-X & $\begin{array}{l}7 \\
7\end{array}$ & 4770 & & $10^{\circ} 1$ & 0,137 & 27 & 4 & $\begin{array}{l}(-58) \\
(-71)\end{array}$ & $\begin{array}{r}61 \\
71\end{array}$ & $\begin{array}{l}1,23 \\
1,57\end{array}$ & 5199 & $\begin{array}{l}429 \\
497\end{array}$ & 12,28 & $\begin{array}{l}1,51 \\
0,92\end{array}$ \\
\hline $12-X$ & 8 & 4273 & & $9^{\circ} 5$ & & & & 42 & $(-42)$ & - & & - & 13,43 & 1,02 \\
\hline $20-X$ & 7 & 4606 & & $8^{\circ} 75$ & & & & $(-144)$ & 144 & 3,51 & & 1008 & 14,22 & 2,12 \\
\hline $\begin{array}{l}\text { 27-X } \\
5-X I\end{array}$ & 9 & $\begin{array}{l}3598 \\
2725\end{array}$ & & $\begin{array}{l}8^{\circ} \\
6^{\circ} 75\end{array}$ & & & & $(-97)$ & 97 & 3,07 & & 873 & $\begin{array}{r}15,13 \\
19,65\end{array}$ & 1,69 \\
\hline
\end{tabular}


$\mu \mathrm{g}$ PS

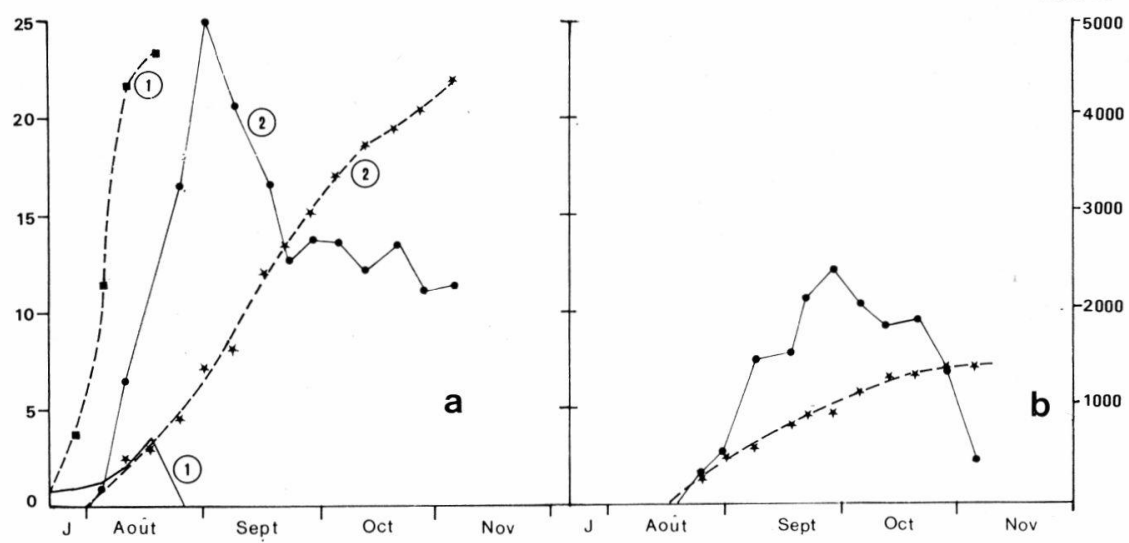

FIG. 22. - Evolution des nombres (trait plein) et des poids moyens (tirets) de Daphnia longispina + (a) et $\sigma^{\circ}(\mathrm{b})$.

1: $q$ issues des éphippies,

2 : $q$ issues des œufs à développement immédiat.

présente un maximum suivi d'un déclin (fig. 22) selon un schéma identique à celui décrit pour $M$. laciniatus.

La diminution des nombres peut s'exprimer par :

$$
\begin{aligned}
& \mathbf{N}_{\mathrm{t}}=\mathbf{N}_{\mathrm{o}} \cdot \mathrm{e}^{-0.0106^{\mathrm{t}}} \text { pour les } \text { o et } \\
& \mathbf{N}_{\mathrm{t}}=\mathbf{N}_{\mathrm{o}} \cdot \mathrm{e}^{-0.0122^{\mathrm{t}}} \text { pour les } \hat{o},
\end{aligned}
$$

soit des taux de mortalité journaliers respectifs de $1,07 \%$ et $1,23 \%$.

Si on considère l'ensemble de la population, la connaissance du nombre d'œufs et du temps de développement d'un œuf permet de calculer l'accroissement théorique de la population entre deux prélèvements successifs. La mortalité correspond à la différence entre l'effectif théorique et l'effectif réel de la population.

Tableau VI. - Durée moyenne $\left(\bar{D}_{i}\right)$ des différentes classes de taille Daphnia longispina + et $\sigma$. La température moyenne du milieu pendant la durée de développement de la classe de taille, a été calculée en tenant compte de la

\begin{tabular}{|c|c|c|c|c|c|}
\hline $\begin{array}{c}\text { Classe } \\
\text { de } \\
\text { taille } \\
(\mathrm{mm})\end{array}$ & $\begin{array}{c}\text { Centre } \\
\text { de } \\
\text { classe }\end{array}$ & $\begin{array}{c}\mathrm{D}_{\mathrm{i}} \\
(\mathrm{j})\end{array}$ & $\begin{array}{l}\mathrm{T}^{\circ} \mathrm{C} \\
\mathrm{D} \stackrel{9}{9}\end{array}$ & $\begin{array}{c}\overline{D_{i}} \cdot \hat{\gamma} \\
(\mathrm{j})\end{array}$ & $\begin{array}{l}\mathrm{T}^{\circ} \mathrm{C} \\
\mathrm{D} \hat{o}\end{array}$ \\
\hline embryon & 0,5 & 1 & & 1 & \\
\hline $0,6-0,8$ & 0,7 & 1,5 & $8^{\circ} 77$ & 1,6 & $11^{\circ} 65$ \\
\hline $0,8-1,0$ & 0,9 & 4,5 & $9^{\circ} 42$ & 5,4 & $11^{\circ} 77$ \\
\hline $1,0-1,2$ & 1,1 & 7,0 & $10^{\circ} 44$ & 17,3 & $10^{\circ} 10$ \\
\hline $1,2-1,4$ & 1,3 & 12,5 & $11^{\circ} 21$ & 26,5 & $9^{\circ} 80$ \\
\hline $1,4-1,6$ & 1,5 & 26,2 & $10^{\circ} 22$ & & \\
\hline $1,6-1,9$ & 1,7 & 49,0 & $9^{\circ} 84$ & & \\
\hline
\end{tabular}
répartition verticale des organismes (cf. fig. 5). 
Elster et Schwoerbel (1970) donnent la durée de développement des œufs de Daphnia longispina en fonction de la température. Ces données ont été reprises pour les calculs présentés dans le tableau V.

Le taux de mortalité journalier, évalué par cette méthode, varie entre 0 et $10,3 \%$. Il est en moyenne de $2,79 \%$ pour l'ensemble de la population, valeur assez proche des taux de mortalité des $\delta$ et des $q(2,30 \%)$.

Les mesures réalisées sur chaque prélèvement nous ont permis de répartir les individus $q$ et $\hat{\alpha}$ par classes de taille de $0,2 \mathrm{~mm}$. L'évolution des nombres dans chaque classe de taille et celle de l'effectif total des ô et des $q$ décrite par les courbes de mortalité, ont été utilisées pour calculer la durée moyenne de développement de chaque classe de taille.

Les résultats sont récapitulés dans le tableau VI.

Banta (1939) a étudié en élevage la durée de développement des différents stades $\&$ de Daphnia longispina. La comparaison des résultats obtenus par cet auteur avec une population faiblement nourrie, et des valeurs calculées «in situ » pour les $\&$ de la population du lac de Port-Bielh, apparaît dans la figure 23. La similitude des courbes de croissance relative montre que les modalités de l'accroissement sont identiques dans les deux populations.

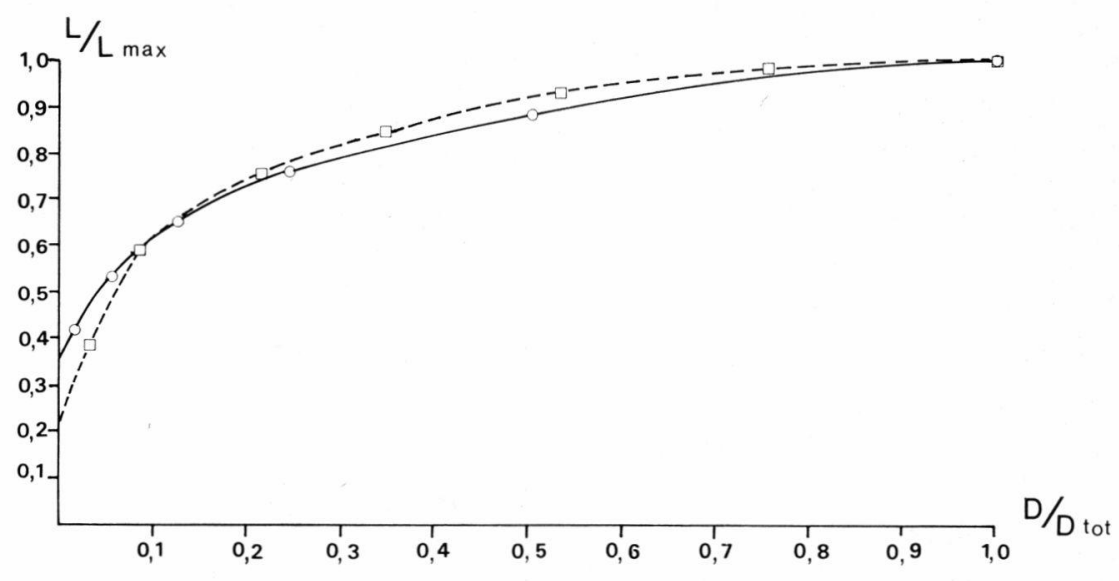

FIG. 23. - Relation entre la longueur relative ( $L / L \max$ ) et la vitesse relative de croissance (D/Dtot) chez les $q$ de Daphnia longispina. En tirets : résultats expérimentaux de Banta (1939); en trait plein : population du lac de Port-Bielh. 


\subsection{2. - Croissance pondérale.}

La biomasse totale et le poids moyen d'un individu $\hat{o}$ et $q$ lors de chaque relevé, ont été calculés à partir de la distribution des tailles des populations $q$ et $\hat{\delta}$, et de la proportion de $q$ ovigères et de $q$ éphippiales dans chaque prélèvement.

Les résultats sont représentés dans la figure 22. Pour les o , nous avons séparé les individus issus des oufs de durée de ceux qui provenaient des œufs à développement immédiat. Deux points sont à retenir de l'examen de ces courbes :

1) La croissance pondérale des $q$ issues des œufs de durée est très rapide. Ces individus atteignent très vite la maturité sexuelle, le nombre moyen d'œufs portés par les $q$ variant de 5 (27.VII ) à 6,6 (10.VIII).

2) Le poids moyen des o et des ô évolue de manière à peu près identique ; il augmente régulièrement jusqu'à un optimum qui se situe le 5 novembre. A cette date, presque tous les individus ont atteint leur taille adulte.

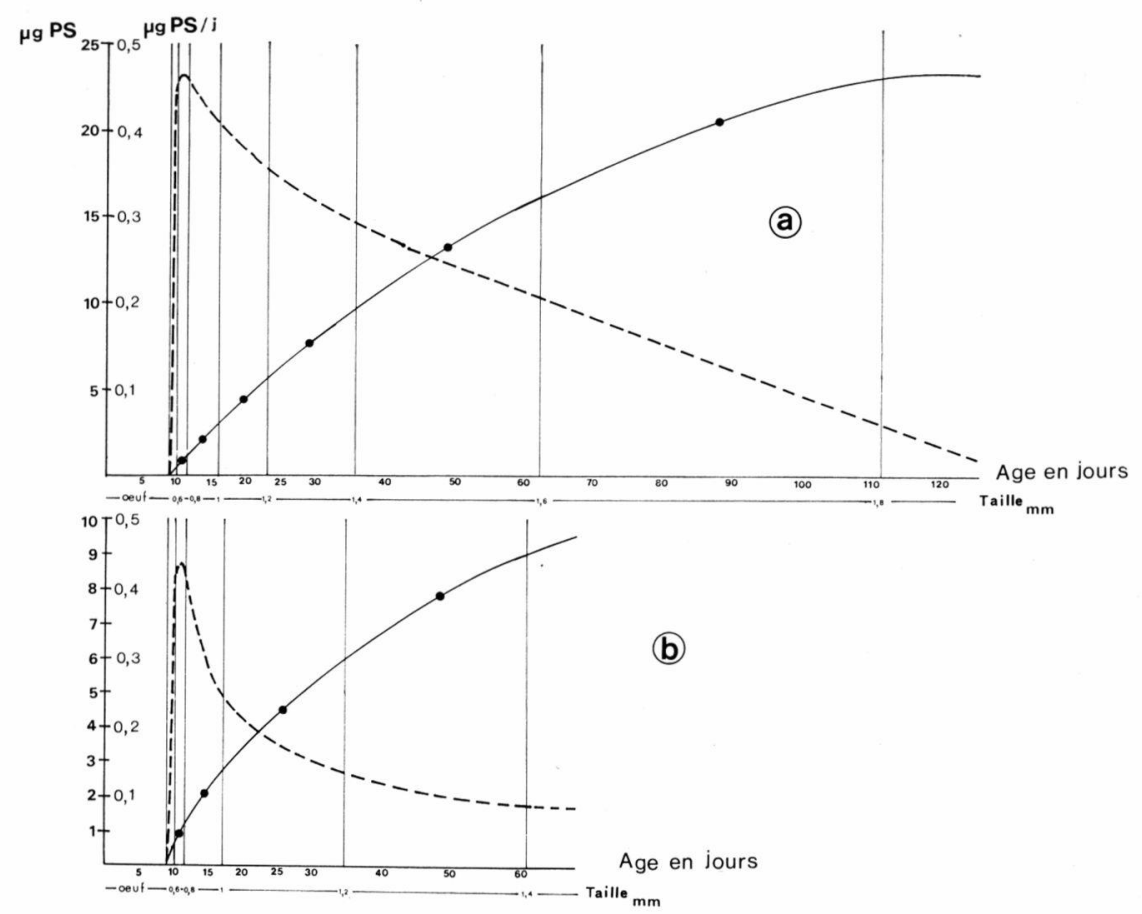

FIG. 24. - Variation du poids individuel (trait plein) et augmentation journalière du poids (tirets) en fonction de l'âge, chez Daphnia longispina ł (a) et $\sigma^{x}(\mathrm{~b})$. 
Nous avons représenté l'évolution du poids des différentes classes de taille en fonction de leur durée de développement (fig. 24 a et b), en considérant que le poids moyen d'un individu d'une classe de taille donnée, se situe au milieu du temps de développement correspondant.

Par différenciation, on peut ainsi calculer l'accroissement journalier du poids en fonction de l'âge.

\subsection{3. - Bromasse et production de Daphnia longispina.}

Elles ont été calculées par les méthodes indiquées pour Mixodiaptomus laciniatus. Nous avons également utilisé les résultats fournis par les calculs de dynamique de population (tableau V), en admettant que la mortalité affecle également tous les représentants de la population. Par suite, la production sera :

$$
\mathbf{P}=\mathbf{N}_{\mathrm{e}} \cdot \overline{\mathrm{p}}+\left(\mathbf{N}_{2} \mathbf{p}_{2}-\mathbf{N}_{1} \mathbf{p}_{1}\right)
$$

où $\mathrm{N}_{\mathrm{e}}=$ nombre d'individus éliminés entre les relevés 1 et 2 ,

$\bar{p}=$ poids moyen d'un individu $=\frac{p_{1}+p_{2}}{2}$,

$N_{1}$ et $N_{2}, p_{1}$ et $p_{2}=$ nombre d'individus et poids moyen d'un individu lors des relevés 1 et 2 .

Les résultats sont donnés dans le tableau VII el les figures 25 et 26 .

La biomasse de Daphnia longispina augmente très rapidement au mois d'août. Elle atteint un maximum à la fin du mois de septembre et se maintient à une valeur à peu près constante pendant tout le mois d'octobre.

La production augmente également au cours du mois d'août. Les valeurs maximales se situent dans la première quinzaine de septembre. Le coefficient $\mathbf{P} / \mathrm{B}$ suit une évolution parallèle. La production annuelle de $D$. Iongispina équivaut à 2,5 fois la biomasse moyenne et à 1,4 à 1,54 fois la biomasse maximale d'octobre. Cette valeur de $\mathrm{P} / \mathrm{B}$ max est encore comparable à celles qui ont élé données pour les larves d'insec'es aquatiques de montagne (opus cit.).

\section{4. - Rotifères.}

Le nombre d'œufs (E) et la durée de développement d'un œuf (D), permettent d'estimer le taux de reproduction $\left(B^{\prime}\right)$.

Parmi les 4 espèces présenles, seul le nombre de $q$ ovigères de Polyarthra vulgaris et Keratella hiemalis a pu être déterminé avec certitude.

Les valeurs de $\mathrm{D}$ ont été déduites des données d'Edmonson (1965) pour Polyarthra vulgaris $\frac{1}{\mathrm{D}}=0,036 \mathrm{~T}-0,021$, pour $\mathrm{T}$ 
Tableau VII. - Estimation de la biomasse et de la production moyennes de Daphnia longispina, d'après l'évolution du poids moyen des individus.

\begin{tabular}{|c|c|c|c|c|c|c|c|c|c|c|c|c|c|}
\hline \multirow[b]{2}{*}{ Date } & \multirow[b]{2}{*}{$\Delta \mathrm{t}$} & \multicolumn{3}{|c|}{$q$ issues des éphippies } & \multicolumn{3}{|c|}{ 우 parthénogénétiques } & \multicolumn{3}{|c|}{$\hat{\circ}$} & \multirow[b]{2}{*}{$\begin{array}{c}\text { Bio- } \\
\text { masse } \\
\left(\mathrm{mg} \mathrm{PS} / \mathrm{m}^{3}\right)\end{array}$} & \multirow[b]{2}{*}{$\begin{array}{l}\text { Produc- } \\
\text { tion } \\
\text { journa- } \\
\text { lière } \\
(\mathrm{mg} \text { PS } \\
\left./ \mathrm{m}^{3} / \mathrm{j}\right)\end{array}$} & \multirow[b]{2}{*}{$\mathrm{P} / \mathrm{B}$} \\
\hline & & $\begin{array}{c}\text { Poids } \\
\text { moyen } \\
\text { d'une } \\
\text { q } \\
(\mu \mathrm{g} P S)\end{array}$ & $\begin{array}{l}\Delta \mathrm{P} / \mathrm{j} \\
(\mu \mathrm{g} \mathrm{PS})\end{array}$ & $\mathrm{N}$ & $\begin{array}{c}\text { Poids } \\
\text { moyen } \\
\text { d'un } \\
\stackrel{+}{+} \\
(\mu \mathrm{g} \text { PS })\end{array}$ & $\begin{array}{l}\Delta \mathrm{P} / \mathrm{j} \\
(\mu \mathrm{g} \mathrm{PS})\end{array}$ & $\mathrm{N}$ & $\begin{array}{c}\text { Poids } \\
\text { moyen } \\
\text { d'un } \\
\hat{o} \\
(\mu \mathrm{g} \mathrm{PS})\end{array}$ & $\begin{array}{l}\Delta \mathrm{P} / \mathrm{j} \\
(\mu \mathrm{g} \mathrm{PS})\end{array}$ & $\mathrm{N}$ & & & \\
\hline 21-VII & & 1,57 & & 169 & - & & - & - & & - & 0,27 & & \\
\hline 27-VII & 6 & 3,60 & 0,339 & 248 & - & - & - & - & - & - & 0,90 & 0,07 & 0,122 \\
\hline 4-VIII & 8 & 11,45 & 0,981 & 128 & 1,11 & - & 184 & - & 一 & - & 1,67 & 0,19 & 0,144 \\
\hline 10-VIII & 6 & 21,73 & 3,727 & 397 & 2,44 & 0,222 & 1263 & - & - & - & 11,71 & 1,14 & 0,170 \\
\hline 17VIII & 7 & 22,36 & 0.090 & 674 & 2,86 & 0,06 & 2351 & - & - & - & 21,80 & 0,16 & 0,009 \\
\hline 24-VIII & 7 & & & & 4,35 & 0,212 & 3310 & 1,57 & - & 300 & 14,87 & 0,60 & 0,032 \\
\hline 1-IX & 8 & & & & 7,22 & 0,359 & 5005 & 2,21 & 0,087 & 550 & 37,36 & 1,53 & 0,058 \\
\hline 8-IX & 7 & & & & 8,38 & 0,165 & 4141 & 3,02 & 0,108 & 1490 & 39,20 & 0,87 & 0,022 \\
\hline 17-IX & 9 & & & & 12,12 & 0,415 & 3281 & 3,98 & 0,106 & 1550 & 45,94 & 1,70 & 0,040 \\
\hline 21-IX & 4 & & & & 13,3 & 0,295 & 2526 & 4,52 & 0,136 & 2115 & 43,15 & 1,10 & 0,025 \\
\hline 28-ilX & 7 & & & & 15,14 & 0,263 & 2758 & 4,60 & 0,010 & 2416 & 52,86 & 0,72 & 0,015 \\
\hline 5-X & 7 & & & & 17,0 & 0,266 & 2734 & 5,87 & 0,181 & 2066 & 58,60 & 1,14 & 0,020 \\
\hline 12-X & 7 & & & & 18,58 & 0,226 & 2441 & 6,57 & 0,282 & 1838 & 57,40 & 0,31 & 0,005 \\
\hline 20-X & 8 & & & & 19.52 & 0,118 & 2716 & 6,61 & 0,006 & 1890 & 65,52 & 0,34 & 0,006 \\
\hline 27-X & 7 & & & & 20,36 & $\begin{array}{l}0,12 \\
0,196\end{array}$ & 2213 & 6,79 & 0,025 & 1385 & 54,46 & 0,48 & 0,009 \\
\hline 5-XI & 9 & & & & 22,13 & 0,190 & 2275 & 7,10 & & 450 & 53,54 & & \\
\hline
\end{tabular}




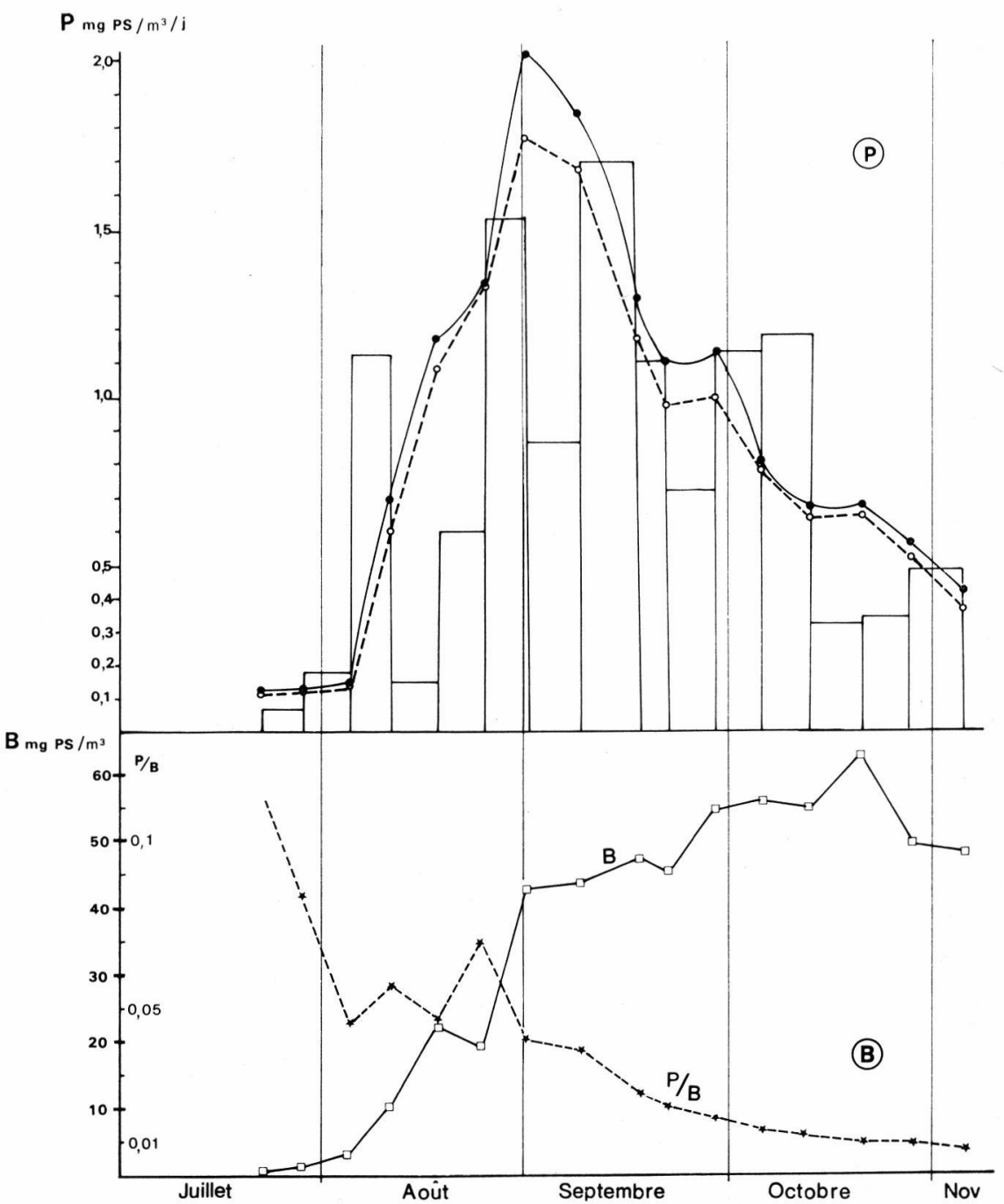

FIg. 25. - Evolution de la porduction moyenne (P), de la biomasse moyenne (B) et du rapport $\mathrm{P} / \mathrm{B}$ chez Daphnia longispina. Les résultats de la production en $\mathrm{mg}$ P.S. $/ \mathrm{m}^{3 / \mathrm{j}}$ correspondent à la méthode 1 a (histogrammes), 2 a (tirets - cercles blancs) et $2 \mathrm{~b}$ (trait plein - cercles noirs).

compris entre $10{ }^{\circ} \mathrm{C}$ et $20^{\circ} \mathrm{C}$ ), et des observations de Nauwerck (1963) pour Keratella hiemalis.

Le nombre d'individus éliminés entre deux prélèvements successifs a été évalué en admettant :

1) une croissance linéaire de la population

$$
N_{e}=\left(B^{\prime} \cdot t \cdot N_{0}\right)-N_{t}
$$


2) une croissance exponentielle de la population. HillbrichtIlkowska (1967) admet que, dans ces conditions, le nombre d'individus éliminés entre 2 relevés successifs correspond à l'effectif

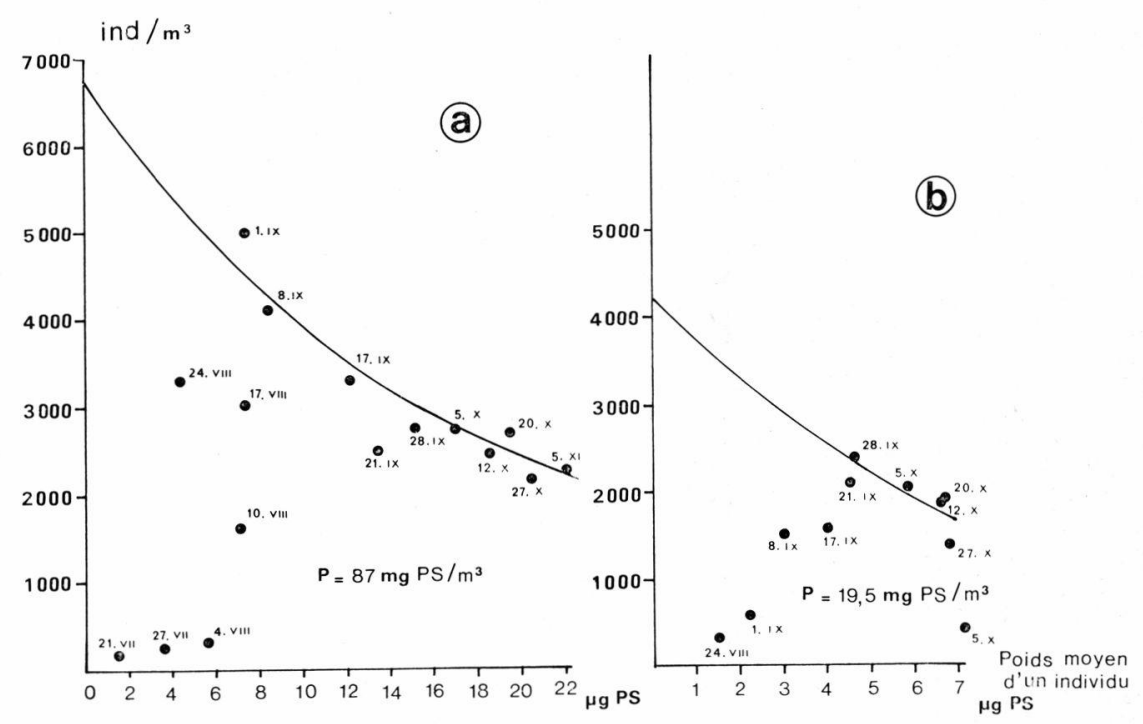

Fig. 26. - Courbe croissance - survie des populations $q$ (a) et $\sigma^{\pi}$ (b) de Daphnia longispina: les courbes représentent l'évolution des nombres estimés d'après les équations de mortalité. La production annuelle moyenne, calculée par intégration des courbes, est indiquée sur la figure.

théorique final-l'effectif final réel. Ce procédé de calcul conduit cependant à une surévaluation de la production puisque la mortalité affecte la population de manière constante. Exprimée par rapport au nombre moyen d’individus présents dans les relevés successifs, la proportion d'individus éliminés quotidiennement est égale à :

$$
\mathrm{M}=\left(\mathrm{B}^{\prime}+1\right)-\mathrm{e}^{\mathrm{r}^{\prime}}
$$

Le poids moyen des Rotifères a été calculé par détermination du biovolume moyen d'un individu en admettant une densité voisine de 1 .

Les tableaux VIII et IX, et la figure 27 donnent les résultats de ces calculs pour Polyarthra vulgaris et Keratella hiemalis.

\section{5. - Comparaison des méthodes d'évaluation de $\mathbf{P}$.}

Les deux méthodes utilisées pour le calcul de la production des Crustacés, reposent sur la détermination :

- de l'augmentation du poids moyen des individus (méthode 1a), 
Tableau VIII. - Dynamique de population et production de Polyarthra vulgaris. $\mathrm{D}_{\mathrm{e}}$ : temps de développement de l'œuf en jours; $\mathrm{E}: \%$ de $q$ ovigères dans la population; $\mathrm{N}:$ nombre moyen d'individus/m $\mathrm{m}^{3} ; \mathrm{b}^{\prime}:$ coefficient de $\mathrm{natalité}=$

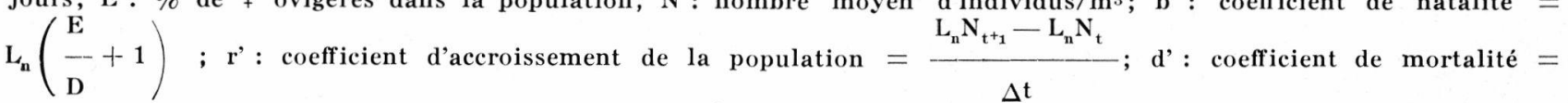
b'- $\mathrm{r}^{\prime} ; \mathrm{M}: \%$ de mortalité journalière $=\left(\frac{\mathrm{E}}{\mathrm{D}}+1\right)-\mathrm{e}^{\mathrm{r} \prime} ; \%$ pertes/jour : nombre d'individus éliminés pour une croissance linéaire; production journalière $=$ accroissement journalier de la population + nombre d'individus éliminés/jour pour une croissance exponentielle(1), et une croissance linéaire (2) de la population.

\begin{tabular}{|c|c|c|c|c|c|c|c|c|c|c|c|c|}
\hline Date & $\Delta \mathrm{t}_{\mathrm{j}}$ & $\mathrm{T}^{\circ} \mathrm{C}$ & $\frac{1}{D_{e}}$ & $\mathrm{E}$ & $\mathbf{N}$ & $\mathbf{b}^{\prime}$ & $\mathbf{r}^{\prime}$ & $d^{\prime}$ & $\mathrm{M} \% / \mathrm{j}$ & $\begin{array}{l}\text { Produc- } \\
\text { tion (1) } \\
\left(\text { ind } / \mathrm{m}^{3} / \mathrm{j}\right)\end{array}$ & $\begin{array}{c}\% \\
\text { pertes } / \mathrm{j}\end{array}$ & $\begin{array}{l}\text { Produc- } \\
\operatorname{tion}(2) \\
\left(\text { ind } / \mathrm{m}^{3} / \mathrm{j}\right)\end{array}$ \\
\hline $2-V I$ & - & $3^{\circ}$ & 0,087 & 一 & 180 & & & & & & & \\
\hline 2-VII & & $3^{\circ} 5$ & 0,105 & 0,397 & 210 & & & & & & & \\
\hline 12-VII & 10 & $5^{\circ}$ & 0,159 & 0,250 & 395 & 0,040 & 0,064 & $(-0,023)$ & 一 & 18 & - & 8 \\
\hline 21-VIII & 9 & $6^{\circ}$ & 0,195 & 1,560 & 2160 & 0,159 & 0,189 & $(-0,030)$ & - & 196 & - & 68 \\
\hline 27-VII & 6 & $7^{\circ} 9$ & 0,264 & 0,809 & 4305 & 0,231 & 0,115 & 0,116 & 13,8 & 826 & 0,07 & 560 \\
\hline 4-VIII & 8 & $9^{\circ} 1$ & 0,307 & 0,978 & 8690 & 0,229 & 0,088 & 0,140 & 16,5 & 1620 & 0,09 & 1107 \\
\hline 10-VIII & 6 & $9^{\circ} 5$ & 0,322 & 0,728 & 9365 & 0,237 & 0,013 & 0,225 & 25,5 & 2414 & 0,25 & 2324 \\
\hline 17-VIII & 7 & $9^{\circ}$ & 0,304 & 0,183 & 13800 & 0,136 & 0,056 & 0,080 & 8,8 & 1959 & 0,07 & 1358 \\
\hline 24-VIII & 7 & $10^{\circ} 5$ & 0,358 & 0,262 & 10500 & 0,073 & $-0,039$ & 0,111 & 11,3 & 1372 & 0,13 & 1031 \\
\hline $1-I X$ & 8 & $9^{\circ} 8$ & 0,333 & 0,183 & 16975 & 0,075 & 0,060 & 0,015 & 1,5 & 1015 & - & 812 \\
\hline 8-IX & 7 & $10^{\circ} 5$ & 0,358 & 0,096 & 29780 & 0,046 & 0,080 & $(-0,035)$ & - & 1829 & - & 808 \\
\hline 17-IX & 9 & $11^{\circ}$ & 0,376 & 0,128 & 12165 & 0,040 & $-0,099$ & 0,140 & 13,6 & 2852 & 0,15 & 1227 \\
\hline 21-IX & 4 & $11^{\circ} 5$ & 0,394 & 0,210 & 9755 & 0,063 & $-0,056$ & 0,119 & 12,0 & 1315 & 0,13 & 795 \\
\hline 28-IX & 7 & $10^{\circ} 9$ & 0,372 & 0,230 & 7750 & 0,080 & $-0,033$ & 0,114 & 11,6 & 1015 & 0,13 & 820 \\
\hline $5-X$ & $\begin{array}{l}7 \\
7\end{array}$ & $10^{\circ} 1$ & 0,343 & 0,432 & 2945 & 0,110 & $-0,139$ & 0,249 & 24,6 & 1315 & 0,30 & 905 \\
\hline $12-\mathrm{X}$ & 1 & $9^{\circ} 5$ & 0,322 & 0,172 & 7755 & 0,098 & 0,139 & $(-0,042)$ & - & 687 & - & 299 \\
\hline $20-X$ & 8 & $8^{\circ} 8$ & 0,295 & 0,299 & 7645 & 0,070 & $-0,002$ & 0,072 & 7,4 & 569 & 0,08 & 557 \\
\hline $27-X$ & 7 & $6^{\circ} 8$ & 9,224 & 0,221 & 12150 & 0,067 & 0,067 & 0,0005 & - & 643 & - & 527 \\
\hline 5-XI & 9 & $5^{\circ} 8$ & 0,186 & 0,709 & 10200 & 0,087 & $-0,019$ & 0,107 & 11,0 & 1227 & 0,12 & 1102 \\
\hline
\end{tabular}




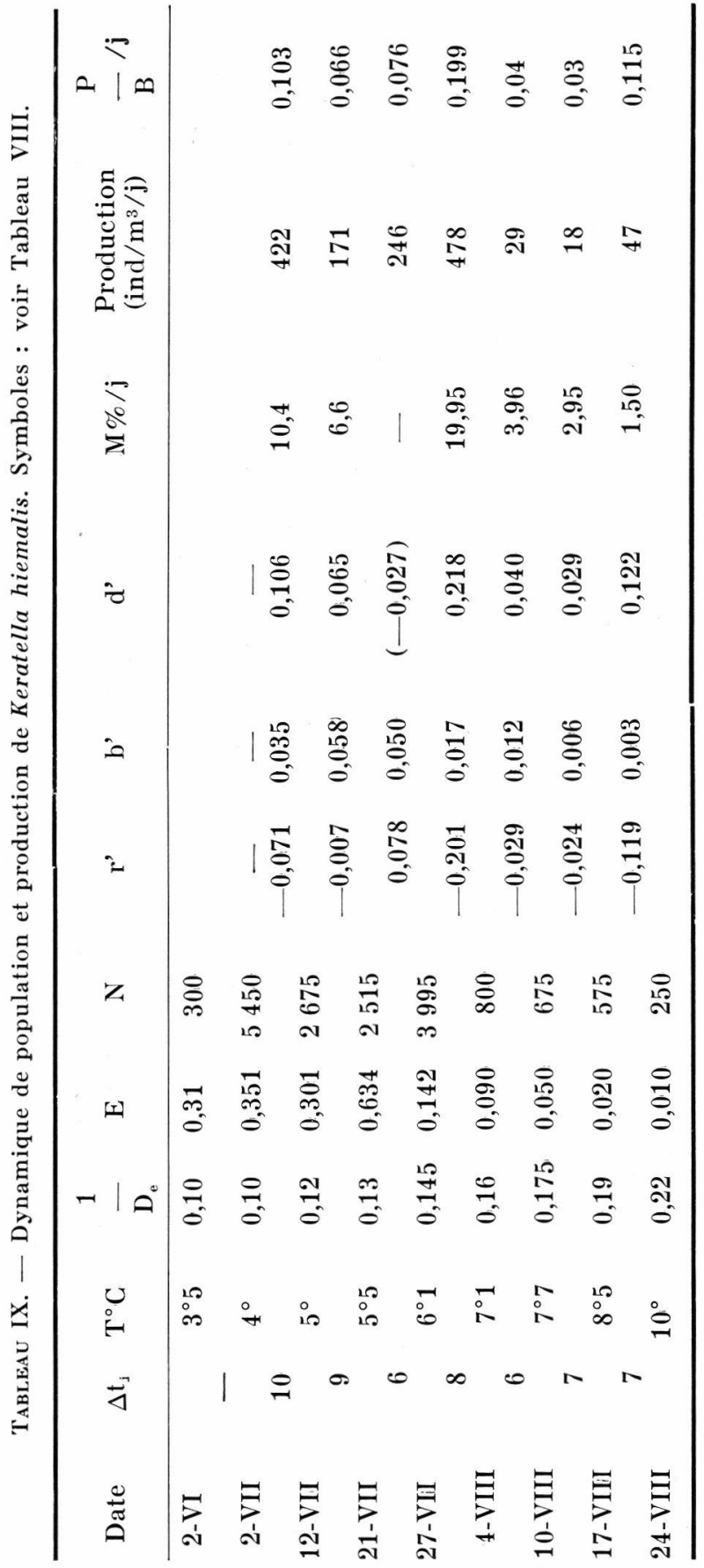




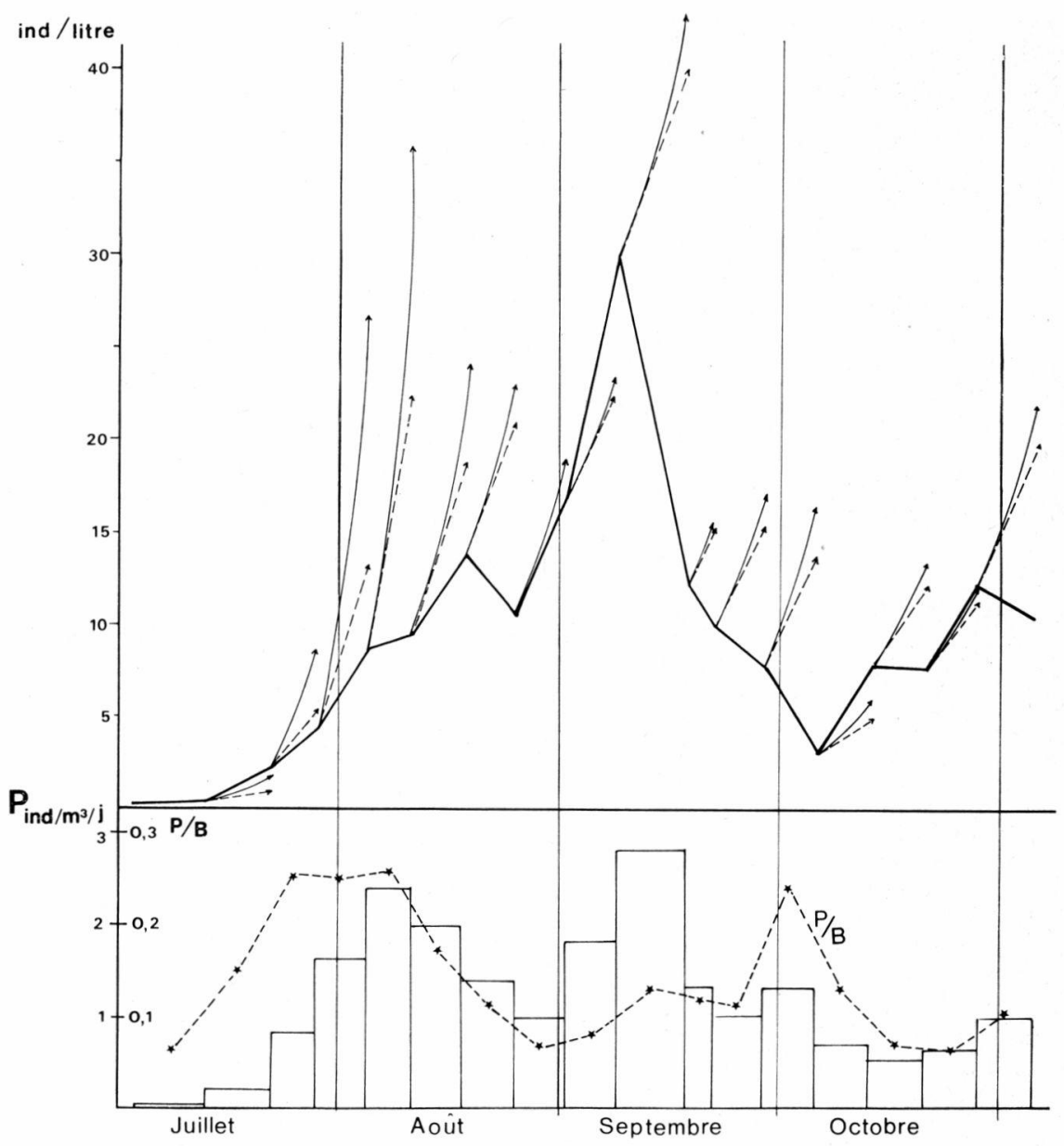

FIG. 27. - Evolution de la densité moyenne (courbe trait plein), de la production (histogrammes) et du rapport P/B (courbe tirets) de Polyarthra vulgais. Les flèches indiquent les effectifs théoriques de la population pour une croissance exponentielle (trait plein) et linéaire (tirets).

- de l'augmentation du poids des individus en fonction de l'âge (méthode 2a).

Les différences avec leur variante graphique :

- courbe croissance - survie (méthode 1b),

- méthode graphique de Winberg et al. (méthode $2 b$ ), tiennent essentiellement à l'arrondi des courbes.

La méthode 1a est plus directement basée sur les valeurs (poids. nombres) otenues lors des échantillonnages. Ceci explique sans doute l'allure plus irrégulière des courbes de production (fig. 21 et fig. 25), 
La mélhode 2a repose sur le lemps de développement (D) des divers stades. Les valeurs de D utilisées ici, correspondent à un lomps de dévelojpement moyen. Or, il est eerlain que D) varie au cours de l'existence d'un stade donné, en fonction de la tempéralure et des conditions de nutrition.

Les deux méthodes donnent cependant des résultats assez voisins (tableau $X$ ). La production annuelle obtenue par la méthode 2a, est supérieure à celle dérivée de la méthode 1 a de $17 \%$ pour M. laciniatus, de $14 \%$ pour D. longispina.

Tableau X. - Comparaison de la production estivale de Mixodiaptomus laciniatus et Daphnia longispina, selon les méthodes d'évaluation :

$$
\begin{aligned}
& 1 a=\sum_{0}^{T}\left(\frac{\overline{P_{2}}-\overline{P_{1}}}{t_{2}-t_{1}}\right) \times\left(\frac{N_{1}+N_{2}}{2}\right) ; \\
& 1 \mathbf{b}=\text { courbe croissance - survic;; } \\
& 2 \mathrm{a}=\sum_{\text {ind }}^{\mathrm{ad} .} \Delta \mathrm{p}_{\mathrm{i}} \times \mathrm{N}_{\mathrm{i}} \text {; }
\end{aligned}
$$

\begin{tabular}{ccc}
\hline $\begin{array}{c}\text { Méthodes } \\
\text { de } \\
\text { calcul }\end{array}$ & $\begin{array}{c}\text { M. laciniatus } \\
\left(\mathrm{mgPS} / \mathrm{m}^{3}\right)\end{array}$ & $\begin{array}{c}\text { D. longispina } \\
\left(\mathrm{mgPS} / \mathrm{m}^{3}\right)\end{array}$ \\
\hline $1 \mathrm{a}$ & 109,1 & 82,4 \\
$1 \mathrm{~b}$ & 113,0 & 106,2 \\
$2 \mathrm{a}$ & 127,6 & 94,2 \\
$2 \mathrm{~b}$ & 132,8 & 100,0 \\
3 & - & 151,3 \\
\hline
\end{tabular}

L'estimation de la production de $D$. longispina à partir du laux de natalité (tableau V), donne des valeurs parfois fort différentes des précédentes. Une source d'erreurs de celte méthode réside dans l'hypothèse que le poids moyen des individus éliminés égale le poids moyen des individus de la population. Ainsi, à la fin du mois d'octobre, la mortalité affecte essentiellemen! la population de $f$ dont l'effectif décroît de $68 \%$, entre le $27 . X$ et le $5 . X I$. Dans ce cas, le poids moyen d'individus éliminés ne correspond pas all poids moyen d'un individu de la population.

D'autre part, en faisant l'hypothèse qu'une proportion d'oeufs égale à l'inverse du temps de développement éclôt chaque jour, on ne tient pas compte du degré de maturité des œufs.

Cette dernière remarque est également valable pour les Rotifères : Edmonson (1965) a montré que les variations du nombre de o matures dans la population pouvaient modifier la valeur du taux de natalité. 


\section{6. - Biomasse et production totale du zooplanton du lac de Port- Bielh.}

Ies diagrammes de distribution verticale (fig. 3-9), montrent que les organismes ne se distribuent pas de manière homogène entre la surface et $Z_{\max }$.

Les résultats précédents doivent donc être corrigés pour tenir compte de la distribution des différentes espèces aux diverses périodes de l'année, et des paramètres morphométriques du lac.

Soient $\mathrm{a}, \mathrm{b}$, c et $\mathrm{d}$, les proportions de la population dans les couches d'eau successives de 0-5 $\mathrm{m}, 5-10 \mathrm{~m}, 10-15 \mathrm{~m}$ et 15-18 $\mathrm{m}$;

$V_{1}, V_{2}, V_{3}, V_{4}$ les volumes respectifs de ces zones,

et $\mathrm{S}$ la superficie du lac.

La production par $\mathrm{m}^{2}$ est donnée par :

$$
\mathrm{P} / \mathrm{m}^{2}=\overline{\mathrm{P}} / \mathrm{m}_{3} \times 18 \times \frac{1}{\mathrm{~S}} \quad\left[\text { a. } \frac{\mathrm{V}_{1}}{5}+\text { b. } \frac{\mathrm{V}_{2}}{5}+\text { c. } \frac{\mathrm{V}_{3}}{5}+\text { d. } \frac{\mathrm{V}_{4}}{3}\right]
$$

Les résultats sont représentés dans les figures $28 \mathrm{a}$ et $\mathbf{2 8 b}$ qui schématisent l'évolution de la biomasse et la production des 3 espèces dominantes du zooplancton du lac de Port-Bielh pendant l'été.

Mixodiaptomus laciniatus constitue la majeure partie de la biomasse totale du zooplancton dont il assure plus de $50 \%$ de la production annuelle. Les Rotifères joulent un faible rôle quantitatif et interviennent ensemble pour moins de $1 \%$ de la biomasse et de la production secondaire pélagique. Minimale à la fin de la période de reproduction de $\boldsymbol{M}$. laciniatus (fin juillet), la biomasse de zooplancton s'accroît progressivement jusqu'à l'automne. La quantité de phyloplanclon présente, dans le lac de Port-Bielh, un maximum fin juillet et de faibles densités d'algues en automne (Capblancq 1972). Cette évolution inverse résulte essentiellement de la consommation des algues par les herbivores qui limitent le développement du phytoplancton de ce lac.

La production annuelle des trois espèces dominantes du zooplancton se situe entre 1,84 et $2,20 \mathrm{gr} \mathrm{PS} / \mathrm{m}^{2} .90 \%$ de cette valeur correspondent aux 5 mois pendant lequel le lac est dégelé (fin juin - fin novembre), le mois d'août représentant à lui seul près de la moitié de la production annuelle.

\section{6. - DISCUSSION}

M. laciniatus, D. longispina et C. strenuus sont les constituants essentiels de la biomasse du zooplancton du lac de Port-Bielh où les Rotifères ( $P$. vulgaris dominant) interviennent pour une faible part. Ces espèces présentent pendant tout leur cycle de développe- 


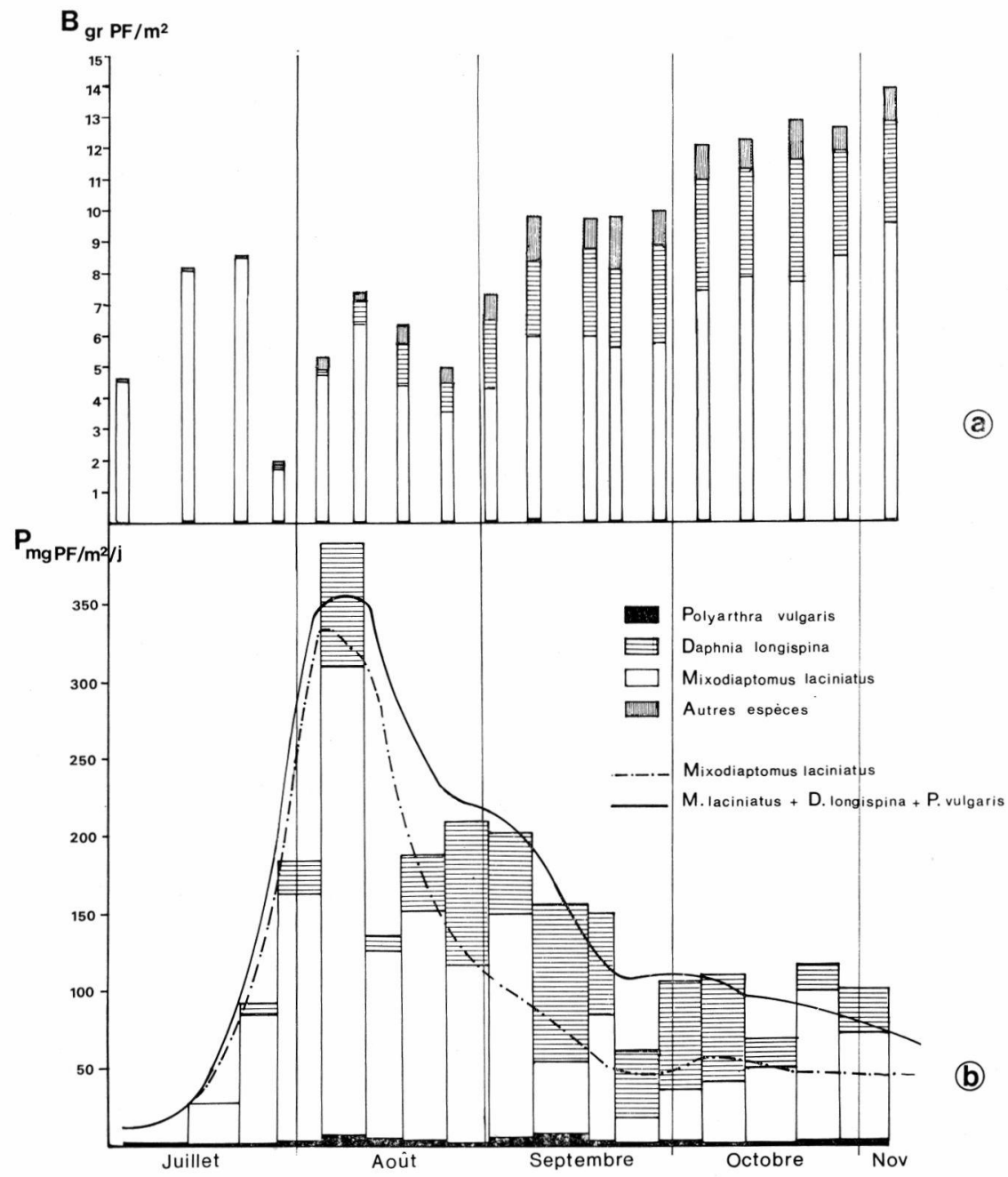

Fig. 28. - Biomasse (a) et Production (b) des espèces dominantes du zooplancton du lac de Port-Bielh. La production des 3 espèces dominantes a été caclulée par la méthode 1 a (histogrammes) et 2 a (courbes).

ment des densités élevées qui contrastent avec les faibles quantités de phytoplancton relevées dans ce lac.

Les Crustacés sont des formes monocycliques. Leur cycle biologique, conforme au schéma général décrit pour les formes benthiques (Rey 1968, Rey et Dupin 1973 b), se caractérise par :

- une longue phase de repos hivernal (novembre - juin), soit sous forme d'œufs de durée (Cladocères), soit à l'état adulte ou subadulte (Copépodes) ; 
- une phase de reproduction intense au dégel (juillel), suivie d'une phase de croissance rabide au milieu de l'été (aoûl-septlembre).

Ce modèle général, qui s’applique également aux larves d'insectes aquatiques (Laville 1972 b, Giani of Laville 1973), paraît essentiellement régi par les conditions thermiques du milieu résultant de l'alternance de deux saisons bien individualisées.

Le rapport $\mathrm{P} / \mathrm{B}$ max est compris entre 1,35 et 1,65 pour $M$. laciniatus, 1,40 et 1,55 pour $D$. longispina. Ces valeurs sont très proches de celles oblenues pour diverses larves d'insectes aquatiques du lac de Port-Bielh (Laville 1972 b, Giani el Laville 1973), et du ruisseau d'Artigusse à $1590 \mathrm{~m}$ (Décamps et Lafont 1973).

Ces résultats, surprenants si l'on considère la diversité des groupes envisagés, reflètent sans doule la similitude des cycles de déreloppement des invertébrés aquatiques de montagne.

Ia biomasse de phyloplancton du lac de Port-Bielh présente un maximum annuel, Irois semaines à un mois après le dégel (Capblaneq 1972). Le rapport des biomasses phytoplancton/zooplancton est alors de 1/0,5 (27.VII.71) à 1/1,7 (4.VIII.71), mais M. lacinialus, élément dominant du zooplancton, se concentre dans les couches superficielles du lac $(0-5 \mathrm{~m})$, landis que les densités algales les plus élevées se rencontrent dans la zone la plus profonde $(12-18 \mathrm{~m})$. En automne, le rapport des biomasses passe de $1 / 9$ (17 et 28 septembre 1971 ) à $1 / 18$ (20 et 27 octobre 1971$)$.

La production primaire du phytoplancton alteint deux maxima en élé (Capblaneq 1972). Le premicr correspond au pic de biomasse de phytoplancton et à la production maximale du zooplancton qu'assure, pour $90 \%, M$. laciniatus ; durant cette période, cetle espòce colonise la zone $0-10 \mathrm{~m}$ où le phytoplanclon peu dense présente les taux de production journaliers les plus élevés. Le second, fin septembre, correspond à l'entrếc du lac en isothermie ; malgré un taux de renouvellement élevé, la biomasse algale reste faible du fait de la prédation par le zooplancton dont la production diminue.

L'évolution inverse des biomasses de phytoplancton el de zooplancton el leur distribution en profondeur, résultent sans doute d'une prédation intense des algues par les consommateurs pélagiques. Celte prédation maintient la densité de phytoplanclon à un faible niveau malgré une activité photosynthétique élevée. Ainsi, la poussée de production primaire automnale n'entraîne pas d'augmentation de la biomasse algale : il existe probablement durant cette période une concurrence alimentaire entre les organismes du zooplancton qui se traduit par une diminution de leur productivilé. La production primaire pélagique, mesurée par la méthode au ${ }^{14} \mathrm{C}$, est de $22,9 \mathrm{~g} \mathrm{C} / \mathrm{m}^{2}$ pour la période allant du dégel au gel $\mathrm{du}$ lac, 
soit 25 à 30 fois celle du zooplanclon. Deux remarques doivent cependant accompagner ce résultal global :

- les calculs de production primaire tiennent compte des pertes de radioactivité lors de la filtration des échantillons, mises en évidence par Arthur et Rigler (1967). Schindler et al. (1972) ont montré récemment que ces perles correspondent à des matières collö̈dales qui, selon eux, « ne peuvent devenir radioactives lorsqu'elles sont mises en incubation avec du ${ }^{14} \mathrm{C}$ minéral dissous en l'absence de plancton ... el doivent par suite être incluses dans les estimations de production », Landis que Mc Mahon (1973) met en doute la validité de cette correction. Si on admet que la production de matériel algal correspond aux valeurs 《non corrigées », clle équivaut à $10,5 \mathrm{~g} C / \mathrm{m}^{2}$ soit 11 à 13 fois celle du zooplancton.

- la méthode au ${ }^{14} \mathrm{C}$ donne une valeur de production qui ne tient pas compte des pertes par respiration nocturne du phytoplancton. Selon Steeman-Nielsen $(1960)$, ees dernières seraient de l'ordre de $40 \%$ de la production journalière. La production du zooplancton représente alors $6 \%$ (valeurs de la production primaire corrigées pour les pertes à la filtration) à $14 \%$ (valeurs non corrigées) de la production primaire pélagique. Ces pourcentages diffèrent peu de ceux que l'on trouve dans les lacs tempérés. Ils semblent indiquer que la production primaire pélagique, uniquement due à des algues nannoplanctoniques, suffit à assurer la nutrition des organismes planctoniques herbivores qui limitent en relour le développement des algues dans le lac.

\section{TRAVAUX GITÉS}

Alben (K. R.). 1951. - The Horokiwi stream. A study of a trout population. Fish. Bull. N.Z., $10: 1-231$.

Amren (H.) . 1964. -... Ecological studies of zooplankton in some ponds on Spitsbergen. Zool. Bid. Ippsala, 36: 161-191.

Angelikr (E.). 1961. -... Les lacs l'Estibère : remarques sur le peuplement des lacs de montagne. Bull. Soc. Hist. Nat. Toulouse, 96 (1/2) : 7-14.

Arthur (C. R.) et Rigler (F. H.). 1967. - A possible source of error in the ${ }^{14} \mathrm{C}$ method of measuring primary productivity. Limnol. Oceanogr., $12: 121-126$.

B.nTA (A. M). 1939. - Studies on the physiology, genetics, and evolution of some Cladocera. Carnegie Instit. Washingt. Paper n*39. Dept of genetics : 1-285.

Bonnet (L.) et CaPhlance (J.). 1973. - Phytoplancton et productivité primaire d'un lac d'altitude dans les Pyrénées. Analyse factorielle des correspondances appliquée aux relevés effectués dans le lac de Port-Bielh. Annls. Limnol., 9 (3) : 183-192.

Capblance (J.). 1972. - Phytoplancton et productivité primaire de quelques lacs d'altitude dans les Pyrénées. Annls. Limnol., 8 : 231-321. 
Capblance (J.). 1973. - Phytobenthos et productivité primaire d'un lac de haute montagne dans les Pyrénées Centrales. Annls. Limnol., 9 (3) : 193-230.

Capblance (J.) et Lavilite (H.). 1968. - Etude morphométrique et physico-chimique de neufs lacs du massif de Néouvielle (Hautes-Pyrénées). Annals. Limnol., 4 :275-324.

Capblance (J.) et Laville (H.). 1972. - Etude de la productivité du lac de Port-Bielh (Pyrénées Centrales). Proc. IBP-UNESCO Symposium on Productivity Problems of Freshwaters, Kazimierz-Dolny, Poland, May 6-12, $1970: 73-88$.

Décamps (H.) et Lafont (M.). 1974. - Cycles vitaux et production des Micrasema pyrénéennes dans les mousses d'eau courante. Annls. Limnol., 10 (1) : 1-32.

Eckstein (H.). 1963. - Untersuchungen über den Einfluss des Rheinwassers auf die Limnologie des Schluchsees. Arch. Hydrobiol., Suppl. $28: 7-182$.

EDMonson (W.T.). 1960. - Reproductive rates of Rotifers in natural populations. Mem. Ist. Ital, Idrobiol., 12 :21-77.

Edmonson (W. T.). 1965. - Reproductive rate of planctonic Rotifers as related food and temperature in nature, Ecol. Monogr., $35: 61-111$.

EichorN (R.). 1957. - Zur Populationsdynamik der Calanoiden Copepoden in Titisee und Feldsee. Arch. Hydrobiol. Suppl. 24, (FalkauSchr. III) : 186-246.

Elster (H. J.). 1954. - Uber die Populationsdynamik von Eudiaptomus gracilis Sars und Heterocope borealis Fischer im Bodensee-Obersee. Arch. Hydrobiol. Suppl, 20, (Falkau-Schr. I) : 487-523.

Elster IH. J.) et SChwoerber (I.). 1970. - Beïtrage zur Biologie und Populationsdynamik der Daphnien im Bodensee. Arch. Hydrobiol. Suppl. 38, 1/2:18-72.

Giani (N.) et Laville (H.). 1973. - Cycle biologique et production de Sialis lutaria L. (Mégaloptère) dans le lac de Port-Bielh (Pyrénées Centrales). Annls. Limnol., $9:$ 45-61.

Giani (N.) et Lucas (C.). 1974. - Les sédiments d'un lac de haute montagne : structure, nature et peuplement. Annls. Limnol. 10 (3) : 223-244.

GouxetTe (P.). 1966. - Les Copépodes des lacs d'Estibère. D.E.S. Toulouse, $35 \mathrm{p}$.

Greze (B. S.). 1963. - Metod rascheta produksiya planktonnykh Kopepod. Zool. Zh., 17 (9) : 1329-1337.

Hillbricht-Illkowska (A.). 1967. - Attempt at evaluation of the production and turnover of plankton Rotifer on the example of Keratella cochlearis (Gosse). Bull. Acad. Pol. Sc. Cl. II XV, $1: 35-40$.

Hillbricht-Ilkowsika (A.) et Patalas (K.). 1967. - Methods of estimating production and biomass and some problems of quantitative calculation methods of zooplancton. Ekologia Polska, Ser. B, XIII, 2 : $139-172$.

Juget (J.) et Giani (N.). 1974. - Répartition des Oligochètes lacustres du massif de Néouvielle (Hautes-Pyrénées) avec la description de Peloscolex pyrenaicus, n. sp. Annls. Limnol., 10 (1) : 33-53.

Kuekowski (R.Z.) et Sushinina (E. A.). 1966. - Energeteskii balans Macrocyclops albidus Jur. v period ego vazvitiya. Ekologii vodnykh organizmov. Moscow : 125-136.

LAville (H.). 1972 a. - Recherches sur les Chironomides (Diptera) lacustres du massif de Néouvielle (Hautes-Pyrénées). I. Systématique, écologie, phénologie. Annls. Limnol., (1971), 7 (2) : 173-332. 
Laville (H.) 1972 b. - Recherches sur les Chironomides (Diptera) lacustres du massif de Néouvielle (Hautes-Pyrénées). IiI. Communautés et production. Annls, Limnol., (1971), 7 (3) : 335-414.

Laville (H.) et Giani (N.). 1974. - Phénologie et cycles biologiques des Chironomides de la zone littorale $(0-7 \mathrm{~m})$ du lac de Port-Bielh (Pyrénées Centrales). Ent. Tidskr. 95 suppl. : 139-155.

Mc MahoN (J. W.). 1973. - Membrane filter retention : a source of error in the ${ }^{14} \mathrm{C}$ method of measuring primary production. Limnol. Oceanogr., $18: 319-324$.

Mordukai-Boltovskoy (F. D.). 1954. - Materialy po srednemu vesu vodnykh bespozvonochnykh bassena Dona. Trudy probl. i Tematich soveshch. ZIN 2 : 223-241.

Nauwerck (A.). 1963. - Die Beziehungen zwischen Zooplankton und Phytoplankton im see Erken. Symb. bol. upsal., 17 (5) : 163 p..

Ness (J. C) et Dugdare (R. C.). 1959. - Computation of production for populations of aquatic midge larvae. Ecology, $40: 425-430$.

Osmera (S.). 1966. - Zur Methode der Festsetzung der Biomasse einiger Plankton Krebstiere. Zool. listy, $15: 79-83$.

Pecen (G. A.). 1965. Produktsiya vetvistonsykh rakoobraznykh ozernogo zooplanktona. Gidrobiol. Zh. 1 (4) : 19-26.

Pourriot (R.). 1965. - Notes taxinomiques sur quelques Rotifères planctoniques. Hydrobiologia, 26 : 579-604.

REY (J.). 1968. - Ecologie des Cladocères du massif de Néouvielle (Hautes-Pyrénées). Annls. Limnol., $4:$ : 325-356.

Rey (J.) et Dupin (B.). 1973 a. - Ecologie des Crustacés benthiques du lac de Port-Bielh (Pyrénées Centrales). I. Répartition. Annls. Limnol., $9:$ 121-134.

Rey (J.) et Dupin (B.) 1973 b. - Ecologie des Crustacés benthiques du lac de Port-Bielh (Pyrénées Centrales). II. Cycles biologiques. Annls. Limnol., 9 : 259-271.

Scherbakov (A. P.). 1952. - Sootnosenic razmerov i vesa u presnovodnych planktonnych rachkov. Dokl. Akad. Nauk SSSR. Gh : 153-157.

Schindeer (D. W.), Schmidt (R. V.) et Reid (R. A.). 1972. -- Acidification and bubbling as an alternative to filtration in determining Phytoplankton production by the ${ }^{14} \mathrm{C}$ method. J. Fish. Res. Bd. Canada, 29 : 1627-1631.

Sushrina (E. A.). 1964. - The rôle of Copépods in the general production of lake zooplancton. Trans. FRBC 1051.

Steeman-Nielsen (E.). 1960. - Productivity of the oceans. Ann. Rev. Plant. Physiol., 11 : 341-362.

Winberg (G. G.). 1971. - Methods for the estimation of production of aquatic animals. Academic Press, London and New York, 175 p. .

Winberg (G. G.), Pecen (G. A.), Sushinina (E. A.). 1965. - Produktsiya Planktonnych rakoobraznykh trekh ozerakh raznogo tipa. Zool. $Z h ., 44: 676-688$.

Winberg (G. G.) et collaborateurs (Ed). 1971. — Symbols, units, and conversion factors in studies of freshwater productivity. IBP Central Office, 7 Marylebone Road, London, $23 \mathrm{p}$. .

Wright (J. C.). 1965. - The populations dynamics and production of Daphnia in Canyon Ferry Reservoir, Montana. Limnol. and Oceanogr., $10: 583-590$. 